\title{
Rab35 Governs Apicobasal Polarity Through Regulation of Actin Dynamics During Sprouting Angiogenesis
}

\author{
Caitlin R. Francis ${ }^{1}$, Hayle Kincross ${ }^{1}$, and Erich J. Kushner ${ }^{1 *}$ \\ ${ }^{1}$ Department of Biological Sciences, University of Denver, Denver, CO \\ *Author for correspondence: \\ Erich J. Kushner \\ University of Denver \\ Department of Biological Sciences \\ Denver, CO 80210 \\ Phone: 303-871-4386 \\ Email: Erich.Kushner@du.edu
}

\begin{abstract}
: 179 words
Manuscript: 5,337 words (excluding methods, citations, figure legends)

Figures: 7

Supplemental Materials: 8 figures, 1 table

Running Title: Rab35 Governs Angiogenic Polarity Programs

Keywords: angiogenesis, lumenogenesis, blood vessel development, actin, Rab35, DENNd1c, trafficking, apical membrane, cytoskeleton, zebrafish
\end{abstract}




\section{ABSTRACT}

36 In early blood vessel development, trafficking programs, such as those using Rab GTPases, are

37 tasked with delivering vesicular cargo with high spatiotemporal accuracy. However, the function

38 of many Rab trafficking proteins remain ill-defined in endothelial tissue; therefore, their relevance

39 to blood vessel development is unknown. Rab35 has been shown to play an enigmatic role in

40 cellular behaviors which differs greatly between tissue-type and organism. Importantly, Rab35

41 has never been characterized for its potential contribution in sprouting angiogenesis; thus, our

42 goal was to map Rab35's primary function in angiogenesis. Our results demonstrate that Rab35

43 is critical for sprout formation; in its absence apicobasal polarity is entirely lost in vitro and in vivo.

44 To determine mechanism, we systematically explored established Rab35 effectors and show that

45 none are operative in endothelial cells. However, we find that Rab35 partners with DENNd1c, an

46 evolutionarily divergent guanine exchange factor, to localize to actin. Here, Rab35 regulates actin

47 polymerization, which is required to setup proper apicobasal polarity during sprout formation. Our

48 findings establish that Rab35 is a potent regulator of actin architecture during blood vessel

49 development. 


\section{INTRODUCTION}

Angiogenesis is the process of sprouting and growth of new blood vessels from preexisting ones and is the primary driver of network expansion [1-4]. Many extrinsic and intrinsic biological systems have been shown to affect endothelial biology and, by extension, blood vessel formation.

54 Membrane trafficking is one such system that is less well-characterized in endothelial tissue, but

55 has recently become more appreciated as additional organotypic trafficking signatures are aligned with important endothelial behaviors [5-8]. Membrane trafficking refers to vesicular transport of protein(s) to, or in vicinity of, the plasma membrane [9-11]. Here, trafficking regulators, such as

58 Rab GTPases, interface with a host of effectors involved in receptor recycling, cytoskeletal

59 regulation, shunting to degradative organelles, lumen formation, basement membrane secretion,

60 and many other signaling events [9, 12, 13]. Indeed, critical to the understanding of how

61 endothelial cells build dynamic and resilient vascular structures is the regulation of membrane trafficking during angiogenic development.

The GTPase Rab35 has been shown to be a multi-faceted regulator of membrane

64 trafficking and continues to be an intensely researched Rab family member [14]. The promiscuity

65 of Rab35 touching multiple pathways has created a cognitive bottleneck in attempting to assign

66 function in any system, due to its seemingly endless diversity of roles. For instance, Rab35 has

67 been shown to be involved in cytokinesis as well as transcytosis of the apical protein podocalyxin

68 during lumen biogenesis in epithelial cysts $[15,16]$. In other investigations, Rab35 has been

69 reported to be a negative regulator of the integrin recycling protein Arf6 via its effector ACAP2

70 [17-19]. Additionally, MICAL1 has been shown to also facilitate Rab35's association with Arf6 and

71 play a role in actin turnover [19-21]. In drosophila, Rab35 regulates apical constriction during

72 germband extension as well as actin bundling via recruitment of fascin $[22,23]$. To date, there is

73 no unified study on Rab35 taking into account its many disparate functions in any tissue.

74 Regarding blood vessel function, no endothelial studies exist detailing how, or if, Rab35 functions 75 in sprouting angiogenesis. 
In the current study, our goal was to comprehensively characterize Rab35's role in

77 sprouting angiogenesis. To do so, we took a holistic approach in investigating established

78 partners of Rab35 and characterized their effect on sprouting behaviors and downstream cellular

79 morphodynamics in vitro and in vivo. Primarily using a 3-dimensional sprouting assay, our results

80 revealed that Rab35 is required for sprouting as its loss significantly disrupts apicobasal polarity.

81 Focusing on Rab35 effectors, we demonstrate that of the many reported effectors only ACAP2

82 was capable of directly binding Rab35 in endothelial cells. However, upon investigating ACAP2

83 and its target Arf6, we determined this established Rab35 trafficking cascade was largely

84 insignificant with regard to sprouting angiogenesis. Excluding all other pathways, we focused on

85 the Rab35 guanine exchange factor (GEF), DENNd1c, and its role in localizing Rab35 to actin

86 structures. Our results demonstrate that DENNd1c facilitates Rab35 tethering to the actin

87 cytoskeleton. Once on actin, Rab35 acts as a positive regulator of actin polymerization and is

88 critical for formation of proper actin architecture. In vivo, we show the requirement of Rab35 in

89 zebrafish blood vessel development using a gene editing approach. Overall, our results provide

90 novel evidence of a focused role for Rab35 as a regulator of actin assembly during sprouting

91 angiogenesis.

\section{MATERIALS AND METHODS}

94 Reagents.

95 All reagent information is listed in the reagents table in the supplementary information.

96

97 Cell Culture.

98 Pooled Human umbilical vein endothelial cells (HUVECs) were purchased from PromoCell and

99 cultured in proprietary media (PromoCell Growth Medium, ready-to-use) for 2-5 passages. All

100 cells were maintained in a humidified incubator at $37^{\circ} \mathrm{C}$ and $5 \% \mathrm{CO}_{2}$. Small interfering RNA

101 (ThermoFisher) was introduced into primary HUVEC using the Neon® transfection system 
102 (ThermoFisher). Scramble, Rab35, Podocalyxin, ACAP2, OCRL, MICAL-L1, DENNd1a,

103 DENNd1b, and DENNd1c were purchased from (ThermoFisher) and resuspended to a $20 \mu \mathrm{M}$

104 stock concentration and used at $0.5 \mu \mathrm{M}$. Normal human lung fibroblasts (NHLFs, Lonza) and

105 HEK-A (ThermoFisher) were maintained in Dulbeccos Modified Medium (DMEM) supplemented

106 with $10 \%$ fetal bovine serum and antibiotics. Both NHLFs and HEKs were used up to 15 passages.

107 For 2-dimensional live-imaging experiments, cells were imaged for one minute at baseline before

108 treatment with CK-666 $(1 \mu \mathrm{M})$, and then imaged for an additional two minutes using 5 second

109 intervals. For ligand-modulated antibody fragments tether to the mitochondria (Mito-LAMA)

110 experiments procedures were carried out as previously described [47]. Briefly, cells were

111 electroporated with mito-LAMA (pCDNA3.0_mitoLAMA-G97), the protein of interest, as well as

112 the target of interest (tag-RFP-DENND1c, mCherry-Arp2, or LifeAct-647). Baseline images were

113 taken for 1 minute (5 second intervals), treated with Trimethoprim (TMP, 500uM) and promptly

114 imaged for an additional 5 minutes at 5 second intervals.

\section{Sprouting Angiogenesis Assay.}

117 Fibrin-bead assay was performed as reported by Nakatsu et al. 2007 [25]. Briefly, HUVECs were 118 coated onto microcarrier beads (Amersham) and plated overnight. SiRNA-treatment or viral 119 transduction was performed the same day the beads were coated. The following day, the EC120 covered microbeads were embedded in a fibrin matrix. Once the clot was formed media was 121 overlaid along with 100,000 NHLFs. Media was changed daily along with monitoring of sprout 122 development. Sprout characteristics were quantified in the following manner. Sprout numbers 123 were determined by counting the number of multicellular sprouts (sprouts that did not contain at 124 least 3 cells were not used in the analysis) emanating from an individual microcarrier beads across 125 multiple beads in a given experiment. Sprout lengths were determined by measuring the length 126 of a multicellular sprout beginning from the tip of the sprout to the microcarrier bead surface across 
127 multiple beads. Percent of non-lumenized sprouts were determined by quantifying the proportion

128 of multicellular sprouts whose length (microcarrier bead surface to sprout tip) was less than $80 \%$

129 lumenized across multiple beads. Sprout widths were determined by measuring the sprout width

130 at the midpoint between the tip and the microcarrier bead across multiple beads. Actin

131 accumulation were defined by actin puncta with a diameter greater than $1.5 \mu \mathrm{m}$. Experimental

132 repeats are defined as an independent experiment in which multiple cultures, containing

133 numerous sprouting beads were quantified; this process of quantifying multiple parameters across

134 many beads and several cultures was replicated on different days for each experimental repeat.

\section{Plasmid Constructs.}

137 The following constructs were procured for this study: GFP-Rab35 S22N inactive (gift from Peter 138 McPherson; Addgene plasmid \# 47426); GFP-Rab35 WT (gift from Peter McPherson, Addgene 139 plasmid \# 47424); GFP_Rab35 Q67L (gift from Peter McPherson, Addgene plasmid \# 47425);

140 mEmerald-Fascin-C-10 (gift from Michael Davidson, Addgene plasmid \# 54094); pARF6(Q67L)-

141 CFP (gift from Joel Swanson, Addgene plasmid \# 11387); pARF6(T27N)-CFP (gift from Joel

142 Swanson, Addgene plasmid \# 11386); pARF6-CFP (gift from Joel Swanson, Addgene plasmid \#

143 11382); pcDNA3-HA-human OCRL (gift from Pietro De Camilli, Addgene plasmid \# 22207);

144 pCDNA3.0_mitoLAMA-G97 (gift from Kai Johnsson, Addgene plasmid \# 130705); pGST1-GGA3-

145 VHS (gift from James Hurley, Addgene plasmid \# 44420); mEmerald-ARP2-C-14 (gift from 146 Michael Davidson, Addgene plasmid \# 53992); MICAL-L1 (Origene, RG214051); and DENNd1c 147 (Origene, RC206410);

149 Lentivirus and Adenovirus Generation and Transduction.

150 Lentivirus was generated by using the LR Gateway Cloning method [24]. Genes of interest and 151 fluorescent proteins were isolated and incorporated into a pME backbone via Gibson reaction 
152 [69]. Following confirmation of the plasmid by sequencing the pME entry plasmid was mixed with

153 the destination vector and LR Clonase. The destination vector used in this study was pLenti CMV

154 Neo DEST (705-1) (gift from Eric Campeau \& Paul Kaufman; Addgene plasmid \#17392). Once

155 validated, the destination plasmids were transfected with the three required viral protein plasmids:

156 pMDLg/pRRE (gift from Didier Trono; Addgene plasmid \# 12251), pVSVG (gift from Bob

157 Weinberg; Addgene plasmid \#8454) and psPAX2 (gift from Didier Trono; Addgene plasmid

158 \#12260) into HEK 293 cells. The transfected HEKs had media changed 4 hours post transfection.

159 Transfected cells incubated for 3 days and virus was harvested.

$160 \quad$ Adenoviral constructs and viral particles were created using the Adeasy viral cloning

161 protocol (9). Briefly, transgenes were cloned into a pShuttle-CMV plasmid (gift from Bert

162 Vogelstein; Addgene plasmid \#16403) via Gibson Assembly. PShuttle-CMV plasmids were then

163 digested overnight with Mssl (ThermoFisher) and Linearized pShuttle-CMV plasmids were

164 transformed into the final viral backbone using electrocompetent AdEasier-1 cells (gift from Bert

165 Vogelstein; Addgene, \#16399). Successful incorporation of pShuttle-CMV construct into

166 AdEasier-1 cells confirmed via digestion with Pacl (ThermoFisher). 5000 ng plasmid was then

167 digested at $37^{\circ} \mathrm{C}$ overnight, then $85^{\circ} \mathrm{C}$ for 10 minutes and transfected in a $3: 1$ polyethylenimine

168 (PEI, Sigma):DNA ratio into 70\% confluent HEK 293A cells (ThermoFisher) in a T-25 flask.

169 Over the course of 2-4 weeks, fluorescent cells became swollen and budded off the plate.

170 Once approximately $70 \%$ of the cells had lifted off the plate, cells were scraped off and spun down

171 at $2000 \mathrm{rpm}$ for 5 minutes in a $15 \mathrm{~mL}$ conical tube. The supernatant was aspirated, and cells were

172 resuspended in $1 \mathrm{~mL}$ PBS. Cells were then lysed by 3 consecutive quick freeze-thaw cycles in

173 liquid nitrogen, spun down for 5 minutes at $2000 \mathrm{rpm}$, and supernatant was added to 2 qty $70 \%$

174 confluent T-75 flasks. Propagation continued and collection repeated for infection of $10-15 \mathrm{~cm}$

175 dishes. After collection and 4 freeze thaw cycles of virus collected from $10-15 \mathrm{~cm}$ dishes, $8 \mathrm{~mL}$

176 viral supernatant was collected and combined with $4.4 \mathrm{~g} \mathrm{CsCl}$ (Sigma) in $10 \mathrm{~mL}$ PBS. Solution

177 was overlaid with mineral oil and spun at $32,000 \mathrm{rpm}$ at $10^{\circ} \mathrm{C}$ for 18 hours. Viral fraction was 
178 collected with a syringe and stored in a 1:1 ratio with a storage buffer containing $10 \mathrm{mM}$ Tris, $\mathrm{pH}$

$1798.0,100 \mathrm{mM} \mathrm{NaCl}, 0.1$ percent BSA, and 50\% glycerol. HUVEC were treated with virus for 16

180 hours at a 1/1000 final dilution in all cell culture experiments.

182 Immunofluorescence and Microscopy.

183 For immunofluorescence imaging, HUVECs were fixed with 4\% paraformaldehyde (PFA) for 7

184 minutes. ECs were then washed three times with PBS and permeabilized with $0.5 \%$ Triton-X

185 (Sigma) for 10 minutes. After permeabilization, cells were washed three times with PBS. ECs

186 were then blocked with $2 \%$ bovine serum albumin (BSA) for 30 minutes. Once blocked, primary

187 antibodies were incubated for approximately 4-24 hours. Thereafter, primary antibodies were

188 removed, and the cells were washed 3 times with PBS. Secondary antibody with $2 \%$ BSA were

189 added and incubated for approximately 1-2 hours, washed 3 times with PBS and mounted on a

190 slide for imaging. For imaging the fibrin-bead assay, first fibroblasts were removed from the clot

191 with a 1-minute trypsin incubation. Following incubation, the trypsin was neutralized with DMEM

192 containing 10\% BSA, washed 3 times with PBS, and fixed using 4\% PFA for 40 minutes. After

193 fixation, the clot was washed 3 times with PBS, permeabilized with $0.5 \%$ Triton-X for 2 hours and

194 then blocked with $2 \%$ BSA for 1 hour prior to overnight incubation with primary antibodies. The

195 following day, primary antibodies were removed, and the clot was washed 5 times with PBS and

196 secondary antibody was added with $2 \%$ BSA and incubated overnight. Prior to imaging the clot

197 was washed 5 times with PBS. All primary and secondary antibodies are listed in the

198 Supplemental Data. Images were taken on a Nikon Eclipse Ti inverted microscope equipped with

199 a CSU-X1 Yokogawa spinning disk field scanning confocal system and a Hamamatusu EM-CCD

200 digital camera. Cell culture images were captured using a Nikon Plan Apo 60x NA 1.40 oil

201 objective using Olympus type F immersion oil NA 1.518. All images were processed using Image J 202 (FIJI). 


\section{Detection of Globular and Filamentous Actin.}

205 Globular and filamentous actin ratios were determined by western blot as described by 206 commercially available G-actin/ F-actin In Vivo Assay Kit (Cytoskeleton). Globular and 207 filamentous immunocytochemistry was performed as previously described [53]. Briefly, cells were

208 fixed with 4\% PFA for 10 minutes and permeabilized in ice cold acetone for 5 minutes and 209 washed. Cells were then incubated for 15 minutes in 2\% BSA with globular actin-binding protein 210 GC globulin (Sigma). Following incubation, cells were washed three times in PBS. After washes 211 cells incubated with an anti-GC antibody in BSA for 15 minutes, washed three times, and 212 incubated in anti-rabbit-555 secondary prior to imaging.

\section{Antibody Feeding Assay.}

215 Antibody feeding assay was carried out as previously described [70]. Briefly, cells were moved to $2164^{\circ} \mathrm{C}$ for 30 minutes to inhibit endocytosis and then $\beta 1$-integrin antibody was added to the culture 217 for an additional 30 minutes. Following incubation, cells were washed 3 times with ice cold PBS 218 and moved back into the $37^{\circ} \mathrm{C}$ degree incubator for 20 minutes. Cells were then fixed with $4 \%$ 219 PFA for 8 minutes and washed with PBS. $\beta 1$-integrin antibody was added once more for 45 220 minutes to label extracellular integrins, washed 3 times with PBS, and then incubated with the 221 secondary antibody (Alexa 555). The secondary was washed 3 times with PBS and then

222 permeabilized with $0.5 \%$ Triton- $X$ for 10 minutes to gain access to the endocytosed $\beta 1$-integrin 223 pool. Then a secondary antibody (Alexa 488) was added for 20 minutes to label the endocytosed 224 integrins, washed and imaged.

\section{Wound Healing Assay.}

227 Treated cells were moved to Ibidi culture insert plates with a two well silicone insert allowing for 228 a defined cell-free gap. At 3 days post siRNA treatment the silicone insert was removed, and 
229 cells were allowed to migrate for 6 hours. Thereafter, cells were fixed, and

230 immunohistochemistry was performed. The distance traveled into the cell free space was

231 measured between groups.

232

233 Immunoblotting \& Protein Pull-Down.

234 HUVEC cultures were trypsinized and lysed using Ripa buffer (20 mM Tris-HCl [pH 7.5], $150 \mathrm{mM}$

$235 \mathrm{NaCl}, 1 \mathrm{mM}$ Na2EDTA, $1 \mathrm{mM}$ EGTA, 1\% NP-40, 1\% sodium deoxycholate, $2.5 \mathrm{mM}$ sodium

236 pyrophosphate, $1 \mathrm{mM} \beta$-glycerophosphate, $1 \mathrm{mM} \mathrm{Na} \mathrm{VO}_{4}, 1 \mu \mathrm{g} / \mathrm{mL}$ leupeptin) containing $1 \mathrm{x}$

237 ProBlock ${ }^{\text {TM }}$ Protease Inhibitor Cocktail -50 (GoldBio). Total concentration of protein in lysate was

238 quantified using the Pierce ${ }^{\mathrm{TM}}$ BCA Protein Assay Kit measured at $562 \mathrm{~nm}$ and compared to a

239 standard curve. $20-50 \mu$ g protein was prepared in 0.52 M SDS, $1.2 \mathrm{mM}$ bromothymol blue, $58.6 \%$

240 glycerol, 75 mM Tris pH 6.8, and 0.17 M DTT. Samples were boiled for 10 minutes, then loaded

241 in a $7-12 \%$ SDS gel and run at $150 \mathrm{~V}$. Protein was then transferred to Immun-Blot PVDF

242 Membrane (BioRad) at $4^{\circ} \mathrm{C}, 100 \mathrm{~V}$ for 1 hour 10 minutes. Blots were blocked in $2 \%$ milk proteins

243 for 1 hour, then put in primary antibody at specified concentrations overnight. After 3 10-minute

244 washes with PBS, secondary antibodies at specified concentrations were applied for 4 hours.

245 After 3 additional PBS washes, blots were developed with ProSignal® Pico ECL Spray.

246 For GGA3 pull-down experiments, GST-GGA3 was grown overnight in $50 \mathrm{~mL}$ of Laria-

247 Bertani broth in NiCo21 E Coli (NEB). The following day the overnight culture was transferred to

$2481 \mathrm{~L}$ of terrific buffer. The culture was monitored for growth and induced at OD600 with IPTG

249 (GoldBio, 12481) at a final concentration of 100uM. Following induction, bacteria were incubated

250 for an additional 3 hours. Induced cells were collected and pelleted, with the pellet resuspended

251 in cold PBS containing $1 \mathrm{mg} / \mathrm{ml}$ lysozyme and $1 \times$ ProBlock $^{\mathrm{TM}}$ Protease Inhibitor Cocktail -50 and

252 then sonicated to lyse bacteria. Cell lysate was clarified by centrifugation and glutathione agarose

253 resin (GoldBio) was added to affinity purify the GST-GGA3. After incubation, agarose resin was

254 washed $2-3$ times with PBS and stored at $-20^{\circ} \mathrm{C}$. 


\section{Zebrafish Transplantation, Microangiography and Gene Editing.}

257 Zebrafish transplantations were performed as previously described [71]. Briefly, cells were 258 harvested at the blastula stage from a tg(kdrl:mCherry) line and treated with CRISPR (described 259 below) line using an Eppendorf CellTram and deposited into recipients harboring a tg(kdrl:eGFP) 260 transgene allowing us to distinguish between host and recipient blood vessels.

For microangiography $48 \mathrm{hpf}$ embryos were (anesthetized) with $1 \%$ tricaine for approximately 20 minutes prior to perfusion. Embryos were then loaded ventral side up onto an

263 injection agarose facing the injection needle. Qdots (ThermoFisher) were sonicated prior to 264 injection. Qdots were loaded into a pulled capillary needle connected to an Eppendorf CellTram 265 and $1-3 \mu$ l of perfusion solution was injected into the pericardial cavity. Once successfully perfused, embryos were embedded in $0.7 \%$ low melt agarose and imaged promptly. Images were taken on a Nikon Eclipse Ti inverted microscope equipped with a CSU-X1 Yokogawa spinning disk field scanning confocal system and a Hamamatusu EM-CCD digital camera using either Nikon Apo LWD 20x NA 0.95 or Nikon Apo LWD 40x NA 1.15 water objective.

Tol2-mediated transgenesis was used to generate mosaic intersomitic blood vessels as

271 previously described $[72,73]$. Briefly, Tol2 transposase mRNA were synthesized (pT3TS-Tol2

272 was a gift from Stephen Ekker, Addgene plasmid \# 31831) [74] using an SP6 RNA polymerase

273 (mMessage Machine, ThermoFisher). A total of 400ng of transposase and 200ng of plasmid 274 vector were combined and brought up to $10 \mu \mathrm{L}$ with phenol red in $\mathrm{ddH} 2 \mathrm{O}$. The mixture was injected 275 into embryos at the 1-2 cell stage. Injected zebrafish were screened for mosaic expression at 48 276 hpf and imaged.

277 CRISPR/cas9-mediated knockouts were performed as previously described [54]. Briefly, 278 equal volumes of chemically synthesized AltR® $\operatorname{crRNA}(100 \mu \mathrm{M})$ and tracrRNAr RNA $(100 \mu \mathrm{M})$ 279 were annealed by heating and gradual cooling to room temperature. Thereafter the 50:50 280 crRNA:tracrRNA duplex stock solution was further diluted to $25 \mu \mathrm{M}$ using supplied duplex buffer. 
281 Prior to injection $25 \mu \mathrm{M}$ crRNA:tracrRNA duplex stock solution was mixed with $25 \mu \mathrm{M}$ Cas9 protein

282 (Alt-R® S.p. Cas9 nuclease, v.3, IDT) stock solution in 20mM HEPES-NaOH (pH 7.5), 350mM

$283 \mathrm{KCl}, 20 \%$ glycerol) and diluted to $5 \mu \mathrm{M}$ by diluting with water. Prior to microinjection, the RNP

284 complex solution was incubated at $37^{\circ} \mathrm{C}, 5 \mathrm{~min}$ and then placed on ice. The injection mixture was

285 micro-injected into 1-2 cell stage embryos. Crispant DNA was retrieved via PCR and subjected to

286 sanger sequencing to visualize indel formation.

288 Zebrafish Live Imaging and Quantification.

289 All zebrafish presented were imaged at $48 \mathrm{hpf}$. Prior to imaging, embryos were treated with $1 \%$

290 Tricaine for 20 minutes and afterwards embedded in $0.7 \%$ low melt agarose. Live imaging of

291 Zebrafish intersomic vessels (ISVs) were performed using the spinning-disk confocal microscopy

292 system mentioned above. ISVs that were analyzed were between the end of the yolk extension

293 and tail. Parameters measured included ISV number, number of non-lumenized vessels (no

294 visible separation between opposing endothelial cells in ISVs), and number of actin accumulations

295 (actin accumulations with a diameter greater than $4 \mu \mathrm{m}$ ).

297 Scanning Electron Microscopy.

298 Cells fixed for SEM were followed the procedure outlined by Watanabe, et al [75]. Scanning 299 electron microscopy was performed at the University of Colorado Anschutz Medical Campus by

300 Dr. Eric Wortchow.

302 Statistical Analysis.

303 Experiments were repeated a minimum of three times. Statistical analysis and graphing were 304 performed using GraphPad Prism. Statistical significance was assessed with a student's unpaired 305 t-test for a two-group comparison. Multiple group comparisons were carried out using a one-way 
analysis of variance (ANOVA) followed by a Dunnett multiple comparisons test. Data was scrutinized for normality using Kolmogorov-Smirnov (K-S) test. Zebrafish sex distribution was not adjusted as sex determination did not occur at the stage of development in which the specimens were assayed. Statistical significance set a priori at $p<0.05$.

\section{RESULTS}

\section{Rab35 is required for sprouting angiogenesis.}

313 To characterize the role of Rab35 in sprouting angiogenesis, we first cloned a fluorescently

314 tagged version of Rab35 into a lentivirus expression system [24]. Thereafter, we transduced ECs

315 and then challenged the cells to sprout in a fibrin-bead assay [25, 26]. Fibrin-bead sprouts

316 demonstrate excellent angiogenic characteristics, reproducing the most salient sprouting

317 behaviors, such as branching, lumenogenesis, anastomosis and tip/stalk cell signaling (Fig. 1A)

318 [7, 8, 27]. Rab35 in 3-dimensional (3D) sprouts demonstrated strong membrane localization, co-

319 localizing with apical marker podocalyxin and luminal actin, opposite basally located $\beta 1$-integrin

320 (Fig. 1B, Movie 1). To test whether Rab35 was necessary for endothelial sprouting, we knocked

321 down Rab35 using siRNA (Fig. 1C). Loss of Rab35 reduced sprout length and sprouts per bead

322 by $\sim 50 \%$, with a significant increase in the percentage of non-lumenized sprouts (Fig. 1D-G).

323 Morphologically, the sprouts appeared stubby, non-lumenized and generally dysmorphic

324 compared with controls (Fig. 1D). These results indicate that Rab35 is required for proper sprout 325 development.

326 Given Rab35 depletion exhibited such a profound impact on sprouting parameters, we 327 stained for various cytoskeletal, apical and basal markers to determine if Rab35 was affecting 328 specific polarity pathways or producing a more global cellular defect. Imaging for VE-cadherin 329 (cell-cell junctions), podocalyxin, $\beta 1$-integrin (basal membrane), moesin (cytoskeletal, apical 330 membrane), synaptotagmin-like protein-2 (apical membrane) and phosphorylated-Tie2 (apical 
331 membrane) revealed that Rab35 knockdown affected all protein localization (Fig. S1A),

332 suggesting that loss of Rab35 globally disturbs cell polarity programs. Emblematic of this was

333 the significant lack of lumen formation and the increase in discontinuous vacuoles in the Rab35

334 depleted condition (Fig. S1B), as lumenogenesis requires proper apicobasal signaling to form [7,

335 28]. We also observed that Rab35 knockdown reduced the number of nuclei per sprout, indicating

336 the presence of cell division defects in line with other reports [16, 29-31] (Fig. S1C). Overall, this

337 data suggests that Rab35 plays a significant role in establishing cell polarity during angiogenic

338 sprouting.

339 We next employed a mosaic approach to determine the cell autonomous nature of Rab35

340 depletion in a sprout collective. To do so, we treated ECs with either Rab35 siRNA or a scramble

341 control. Thereafter, the knockdown population was marked with cell-tracker and mixed 50:50 with

342 wild-type ECs. The resulting mosaic sprouts contained a mixture of siRNA-treated and untreated

343 ECs (Fig. 1H). Cells contained within sprouts were then binned into two categories: 1) not-

344 opposing, an isolated siRNA-treated cell; or 2) opposing, two adjacent siRNA-treated ECs (Fig.

345 1I,J). Our results demonstrate that Rab35 knockdown in not-opposing ECs contained actin-

346 labeled vacuolations and polarity defects as indicated by a reduction in lumen formation compared

347 with scramble-treated controls (Fig. 1I-M). For Rab35 depleted ECs in the opposing orientation

348 defects were even more pronounced with complete lumen failures at these sites, while also

349 exhibiting multiple vacuolations and polarity defects (Fig. 1I-M). Overall, these results indicate

350 that Rab35 is cell autonomous and is required for EC polarity.

352 Rab35 resides at the apical membrane during sprouting.

353 As the loss of Rab35 produced such a profound effect on EC sprouting, we sought to

354 better understand its cellular localization to gain insight into its potential function. In sprouts,

355 quantification of Rab35 enrichment between different cellular compartments showed a preference

356 for the apical membrane for wild-type (WT) and constitutively active (CA) Rab35 variants, while 
357 the dominant-negative (DN) Rab35 mutant resided in the cytoplasm (Fig. 2A). In this regard, 358 subcellular imaging of WT and CA Rab35 showed a strong colocalization with apical podocalyxin

359 (Fig. 2B). Similar to loss of Rab35, expression of the DN Rab35 also produced polarity defects, 360 such as mislocalization of podocalyxin and large actin accumulations (Fig. 2B). To more 361 conclusively assign Rab35 phenotypes, we performed several rescue assays by knocking down 362 the endogenous Rab35 and then over-expressing Rab35 variants in sprouts. Expression of WT 363 or CA Rab35 decreased the number of non-lumenized sites in sprouts compared to endogenous

364 Rab35 knockdown alone expressing a GFP control, but not to levels in the scramble treated group

365 (Fig. 2C,D; S2A,B). Rab35 knockdown and expression of the DN Rab35 mutant showed the 366 highest increase in dysmorphic sprouts, exhibiting numerous accumulations of actin puncta and 367 lumen defects, again suggesting Rab35 is necessary for sprout function. described [16, 29, 31], but had no preference for filopodia extensions or tip-cell positioning (Fig. S3A,B). In 2D culture, we also observed that Rab35 modestly colocalized with filamentous actin

371 in a monolayer; however, this association was reduced in migratory cells (Fig. S3C,D). Previous 372 reports have implicated Rab35 in Wiebel Palade Body (WPB) granule release [32]. Although loss 373 of Rab35 may alter WPB secretion, likely due to the impact on cell polarity, in our hands Rab35 374 did not colocalize with these structures in 2D or 3D culture systems (Fig. S3E,F). These results 375 indicate that Rab35 is largely localized to the apical membrane in its active form as well as areas 376 of high actin density. 
383 to the apical membrane during lumenogenesis, we overexpressed TagRFP-Rab35 and stained

384 for endogenous podocalyxin in 2D culture and did not detect any significant signal overlap (Fig.

385 S4A). Previous literature showed that Rab35 directly binds to the cytoplasmic tail of podocalyxin

386 [16]. Overexpression of the human podocalyxin cytoplasmic domain (residues 476-551) and

387 TagRFP-Rab35 also did not show any obvious association (Fig. S4A). To further probe for this

388 previously reported binding between Rab35 and podocalyxin, we engineered a mitochondrial-

389 targeted Rab35 to test what proteins or complexes bind Rab35 and are then 'pulled' along to

390 mitochondria. Expression of WT or CA mitochondrial-targeted Rab35 did not show any

391 association with endogenous podocalyxin or overexpression of its cytoplasmic tail domain (Fig.

392 S4B,C). We next reasoned if mistrafficking of podocalyxin by way of Rab35 depletion was the

393 predominant mechanism underpinning the sprouting defects, then knocking down podocalyxin

394 would produce a similar phenotype as compared with loss of Rab35. Knockdown of podocalyxin

395 did not phenocopy Rab35-mediated sprouting defects (Fig. S4D-I). The only exception was that

396 podocalyxin knockdown increased the percentage of non-lumenized sprouts compared with

397 controls. Overall, our data suggests that Rab35 does not directly participate in podocalyxin

398 trafficking in ECs; however, loss of Rab35 distorts podocalyxin's localization to the apical

399 membrane likely due to other alterations in cell polarity.

\section{Rab35 interacts with ACAP2 in endothelial cells.}

To take a more holistic approach in determining how Rab35 functions in endothelial tissue,

403 we performed a functional screen by knocking down the most highly cited Rab35 effectors singly

404 and in combination, to determine if any effector combination phenocopied Rab35 sprouting

405 defects (Fig. 3A,B) [15, 17-19, 30, 32-35]. First, we found that Rab35 itself did not produced a

406 significant effect on 2D cell motility, suggesting the primary defect in sprouting may be due to

407 altered apicobasal polarity only detectable in the 3D sprout environment (Fig. S5A,C). As Rab35

408 and ACAP2 have been shown to affect the integrin recycling pathway via their association with 
Arf6, we also assayed for integrin recycling as defective integrin signaling could also affect cell

410 polarity. As compared with the scramble controls, knockdown of Rab35 and OCRL significantly

411 increased integrin recycling, while ACAP2 and MICAL-L1 had no effect (Fig. S5B,D).

412 Next, we determined that RUSC protein levels were not detectable in ECs, thus was

413 excluded from our screen (Fig. S6F). ACAP2, OCRL, or MICAL-L1 or any combination of

414 knockdown targeting these proteins, demonstrated the greatest phenotypic similarity to Rab35

415 knockdown with regard to sprouting parameters (Fig. 3C-G). Upon closer inspection, both ACAP2

416 and OCRL knockdowns were associated with elevated frequencies of non-lumenized sprouts with

417 disorganized actin, although to a lesser extent than compared with Rab35 (Fig. 3C). These results

418 suggest that ACAP2 and OCRL potentially resemble a Rab35 sprouting defect.

419 Both ACAP2 and OCRL have been reported to directly bind Rab35 [17-19, 30, 32, 35];

420 however, this interaction has not been validated in ECs. First, we overexpressed tagged versions

421 of ACAP2, OCRL and MICAL-L1 to visualize their localization patterns with Rab35 in ECs. Rab35

422 and ACAP2 strongly colocalized to the plasma membrane, while Rab35 did not show strong

423 localization with ORCL or MICAL-L1 (Fig. 3H). Further testing for potential interactions, we again

424 used the mitochondrial-targeted Rab35 to visualize any physical association between Rab35 and

425 these previously published effectors. Co-expression of WT and CA Tom20-Rab35 with ACAP2

426 demonstrated strong colocalization at the mitochondria, while the DN Rab35 showed no

427 significant binding of ACAP2 (Fig. 3I; S6A,B). We performed this same experiment using ACAP2

428 with the ankyrin repeat domain deleted and observed no binding, indicating Rab35 directly

429 interacts with this domain (Fig. S6B). As a control, we also co-expressed a tom20-Rab27a and

430 ACAP2 and observed no mislocalization of ACAP2 (Fig. S6C), suggesting ACAP2's affinity for

431 Rab35 is specific. Co-expression of WT, CA or DN Tom20-Rab35 with OCRL or MICAL-L1 did

432 not show any colocalization at the mitochondria, signifying a lack of binding (Fig. 3I; S6D,E).

433 These results demonstrate that ACAP2, not OCRL or MICAL-L1, directly interacts with Rab35 in 434 endothelial tissue. 
Rab35 does not impact Arf6 activity in endothelial cells.

Previous literature has shown that ACAP2 works as a GTPase activating protein (GAP) with Rab35 to inactivate the GTPase Arf6 [17, 18, 33]. Arf6 has been shown to be involved with actin remodeling and integrin recycling [19, 36-38]. To test if this association exists in ECs, we

440 first determined the localization of Arf6 relative to Rab35 and ACAP2 in 2D culture. Cells 441 expressing tagged Rab35 and Arf6, or ACAP2 and Arf6 demonstrated modest colocalization 442 throughout the cell with the greatest colocalization at the cell cortex (Fig. S7A). Using WT, CA 443 and DN versions of Arf6, we stained for actin to determine if Arf6, like Rab35, associated with 444 actin structures. Similar to Rab35, in peripheral membrane protrusions, WT and CA Arf6 445 demonstrated moderate colocalization with actin; however, this association did not persist on 446 filamentous actin located towards the cell interior (Fig. S7B). In sprouts, Arf6 showed weak 447 localization to the apical membrane as compared to Rab35 (Fig. S7C). Once again using 448 mitochondrial-mistargeting, we tested for binding between Arf6 and Rab35. Mitochondrial449 targeted Rab35 did not pull Arf6, indicating a lack of binding interaction (Fig. S7D). To further 450 confirm this, we used the Tom20 epitope to target ACAP2 to the mitochondria to determine if Arf6 451 interacts with ACAP2. Our results show that Arf6 does not localize to the mitochondria, indicating 452 ACAP2 does not strongly interact with Arf6 in ECs (Fig. S7D). We reasoned that the lack of 453 binding between Arf6 and ACAP2 could be due to an insufficiency of Rab35, as Rab35 is 454 hypothesized to regulate ACAP2's availability to act upon Arf6 [17]. Therefore, we simultaneously 455 expressed Tom20-Rab35, TagRFP-ACAP2 and HA-Arf6 in hopes that the Rab35 bound to 456 ACAP2 would recruit Arf6 to the mitochondria. Our results demonstrate that Arf6 did not localize 457 with mitochondrial Rab35 and ACAP2, suggesting ACAP2 does not directly act upon Arf6, or that 458 this signaling does not require a robust binding interaction in ECs (Fig. S7E).

459 Due to the wealth of literature demonstrating loss of Rab35 increases Arf6 activity in non460 endothelial tissues, we sought to confirm this signaling interaction biochemically. To do so, we 
461 first expressed WT, CA, and DN versions of Arf6 in ECs and used recombinant GGA3 to pulldown

462 the active form of Arf6 as others have reported [39]. Pulldown using GGA3 demonstrated more

463 binding with the CA mutant as compared with the WT and DN versions of Arf6, validating this

464 approach for testing Arf6 activity (Fig. S7F). Next, we knocked down and over-expressed Rab35

465 in ECs and then probed for active Arf6. Knockdown of Rab35 or overexpression of Rab35 did not

466 significantly alter Arf6 activity in ECs (Fig. S7G). These results would suggest that loss of Rab35

467 does not affect Arf6 activation. Previous literature reported that knockdown of Rab35 promoted

468 Arf6 activity; thus, we next tested if overactivation of Arf6 would phenocopy the Rab35 loss of

469 function sprouting phenotype to more thoroughly factor out this signaling pathway. Moving to Arf6

470 overexpression in sprouts, we observed that both WT and CA Arf6 marginally affected sprouting

471 parameters with the WT and CA Arf6 increasing the frequency of lumen failures compared with

472 the DN version (Fig. S7H). A primary phenotype in sprouts deficient in Rab35 was abundant actin

473 aggregates and the presence of non-apical podocalyxin. Inconsistent with these observations,

474 ECs expressing CA Arf6 demonstrated normal actin architecture as well as typical podocalyxin

475 apical deposition (Fig. S7I). These results suggest that overactivation of Arf6 due to loss of Rab35

476 is likely not the causative pathway promoting sprouting defects.

DENNd1c is required for Rab35 function.

We were intrigued by the idea that other roles of Rab35 were being unaccounted for as Arf6 activation was largely unaffected by loss of Rab35 in ECs. Earlier we observed that Rab35

481 colocalized with actin. Additionally, a consistent phenotype we observed was impaired actin 482 organization, marked by actin aggregates in Rab35 knockdown sprouts. To this end, Rab35 has 4833 GEFs, DENNd1a-c [40-43]. DENNd1c has been shown to play a more uncharacteristic role, 484 being less involved with GTP hydrolysis, but demonstrating the lone ability to bind to both globular 485 and filamentous actin, mediating Rab35 localization to these microfilaments [41]. Exploring this 486 association, we knocked down DENNd1a-c individually and in combination. Loss of DENNd1a 
487 and DENNd1b did not produce any significant impact on sprouting morphology; however,

488 knockdown of DENNd1c alone resulted in growth of dysmorphic sprouts mirroring Rab35 loss of

489 function (Fig. 4A-E; Movie 2,3). Knockdown of all DENNd1s produced the greatest effect on

490 sprouting behaviors, presumably because the GEF activity provided by DENNd1a/b was also lost

491 (Fig. 4D-F). We also confirmed that knocking down any given DENNd1 did not result in a

492 compensatory increase in expression of the remaining DENNd1s (Fig. S8B). Staining for actin

493 demonstrated that DENNd1c knockdown produced the greatest number of aberrant actin

494 accumulations similar to the Rab35 knockdown phenotype (Fig. 4C,G). We next cloned and

495 tagged DENNd1c to visualize its cellular localization with Rab35. DENNd1c and Rab35 showed

496 strong colocalization on actin in 2D cell culture (Fig. S8A). We also expressed Rab35 with the

497 integral actin protein Arp2 that mediates actin filament branching [44], Rab35 localized to areas

498 of active polymerization marked by Arp2/Rab35 localization. Rab35, Arp2 and filamentous actin

499 colocalized in many areas (Fig. S8A). To explore if DENNd1c, per se, was responsible for

500 tethering Rab35 to actin, we individually knocked down all three DENNd1s and quantified the

501 relative amount of Rab35 uniformly localized at the plasma membrane, accumulated at the

502 plasma membrane or in the cytoplasm. DENNd1c knockdown exhibited the greatest increase in

503 apical plasma membrane accumulations compared with DENNd1a or DENNd1b (Fig. 4H). These

504 data indicate that loss of DENNd1c phenocopies the Rab35 knockdown effect on sprouting

505 parameters and the actin cytoskeleton.

507 Rab35 and DENNd1c localize to sites of actin polymerization.

508 We next sought to comprehensively characterize the association between Rab35,

509 DENNd1c and branched actin located at the cell periphery. To do so, we again overexpressed

510 the actin-specific protein Arp2 as a marker of active actin polymerization [45]. Both Rab35 and

511 DENNd1c demonstrated strong colocalization to Arp2 and the underlying actin (Fig. 5A). To

512 specifically perturb the branched actin network, we next treated cells with the Arp2/3 inhibitor CK- 
513666 [46] and then determined the effect on Rab35 and DENNd1c localization. In 3D sprouts,

514 inhibition of branching actin resulted in accumulations of actin similar to the Rab35 knockdown

515 phenotype (Fig. 5B, S8D; Movie 4,5). In 2D culture, CK-666 treatment rapidly depleted actin at

516 the cell cortex (Fig. 5C). Rab35 prior to CK-666 administration exhibited a uniform distribution in

517 the plasma membrane with enrichment at sites of actin accumulation adjacent to the cell

518 periphery. However, after inhibition of branched actin formation Rab35 collapsed into discrete

519 puncta scattered throughout the cytoplasm. Interestingly, CK-666 treatment created large,

520 presumably globular, actin vacuoles which were then surrounded by Rab35 (Fig. 5C, S8E; Movie

521 6); we believe these structures are analogous to the actin accumulations observed in sprouts

522 when Rab35 is depleted. As a control we performed the same experiment with Rab11a and did

523 not observe any alteration in Rab11a localization with CK-666 treatment (Fig. S8C), suggesting

524 not all Rabs are dependent on actin for their localization. Using the same approach with

525 DENNd1c, we observed, again, DENNd1c was highly enriched at cortical actin; however,

526 treatment with CK-666 effectively depleted DENNd1c from this actin population (Fig. 5D). Unlike

527 Rab35, CK-666 treatment did not cause the formation of puncta, but the redistribution of

528 DENNd1c to unaffected actin, such as filamentous actin towards the cell interior (Fig. 5D; Movie

529 7). As a control, we treated cells with CK-666 expressing both Rab35 and Arp2. As expected,

530 Arp2 was no longer located on actin, collapsing into puncta, while remaining adjacent to Rab35

531 (Fig. 5E; Movie 8). These data suggest that Rab35 and DENNd1c are recruited to polymerizing

532 actin filaments. Additionally, in the absence of active actin polymerization Rab35 collapses into

533 vesicular structures.

534 To visualize Rab35's temporospatial recruitment to cortical actin, we employed a 535 chemically switchable GFP-binding nanobody, termed ligand-modulated antibody fragments 536 (LAMAs) [47]. This method allowed us to sequester GFP-tagged Rab35 at the mitochondria and 537 then rapidly release the protein upon drug treatment, enabling dynamic imaging of localization 538 patterns (Fig. 5F). Using GFP-Rab35, LAMA and TagRFP647-LifeAct [48] expressing cells, we 
release GFP-Rab35 from mitochondria and live-imaged its localization preferences. Our data shows that Rab35 quickly localizes to the cell periphery following trimethoprim (TMP) treatment.

541 Of note, Rab35 did not co-localize with longer-lived filamentous actin, as the LifeAct probe 542 primarily decorates this population (Fig. 5G, Movie 9). When repeated with tagged Arp2, Rab35 543 quickly ( $2 \mathrm{~min}$ ) localized to Arp2 puncta on the cell cortex (Fig. 5H; Movie 10). Rab35 also 544 demonstrated a preference for sites of DENNd1c when released from the mitochondria (Fig. 5I,

545 Movie 11). Next, we released Rab35 and imaged its localization to Arp2, and then immediately 546 treated with CK-666 to determine how this association would be affected. Administration of CK547666 rapidly dissociated Rab35 and Arp2 at the cortex (Fig. 5J; Movie 12). Lastly, to test if 548 DENNd1c was responsible for recruiting Rab35 to branched actin, we knocked down DENNd1c 549 and repeated the LAMA localization experiments. Upon release, Rab35 showed a reduction in its 550 ability to localize to cortical Arp2, suggesting DENNd1c is important for this interaction (Fig. 5K;

551 Movie 13). Overall, these data suggest that Rab35 is rapidly recruited to the cortex and is 552 anchored to actin filaments by DENNd1c.

Rab35 promotes actin assembly.

Our previous data indicates that in the absence of Rab35 global apicobasal polarity is

556 affected, which is likely due to significant alterations in the actin cytoskeleton. Also, Rab35 557 colocalized with actin and actin polymerizing protein Arp2. Thus, our next aim was to test whether 558 Rab35 affected actin polymerization, per se. Prior literature indicates that Rab35 would increase 559 actin polymerization via its purported trafficking interactions with Cdc42 and Rac1 [40, 41, 43]; 560 however, others have claimed Rab35 may act as a brake for actin polymerization through its 561 association with MICAL-L1 in non-endothelial tissues [21].To begin to explore how Rab35 impacts 562 actin in ECs, we transfected Rab35 variants WT, CA and DN into freely migrating ECs. It is well563 established that lamellipodia protrusions (membrane movement away from the cell body) and 564 retractions (membrane movement towards the cell interior) are primarily mediated by local actin 
565 assembly and disassembly $[49,50]$. Attempting to monitor global lamellipodia dynamics in an

566 unbiased fashion, we employed the open source software ADAPT [51]. Our analysis determined

567 that only the Rab35-CA mutant significantly increased both the cells protrusive and retractive

568 capabilities, a finding in line with enhanced migration (Fig. 6A,B). Interestingly, knockdown of

569 Rab35 did not shift membrane dynamics significantly, potentially suggesting Rab35-based actin

570 regulation may play a more critical role in $3 \mathrm{D}$ sprouting.

571 Based off this finding, we reasoned that if Rab35 was involved with actin polymerization,

572 then knockdown of Rab35 would shift the balance between globular and filamentous actin to skew

573 more globular, as less filaments are being assembled. Using differential centrifugation, we

574 separated the globular and filamentous pools of actin as previously reported [52]. Rab35

575 knockdown significantly increased the globular actin abundance compared with control (Fig.

576 6C,D). Using a similar method, we stained for globular actin using GC globulin and phalloidin to

577 detect the filamentous actin [53]. Again, our results demonstrated an increase in globular to

578 filamentous actin ratio in the absence of Rab35 as compared with controls (Fig. 6E,F). We also

579 co-stained for globular and filamentous actin while expressing Rab35 to ensure Rab35

580 colocalized with both actin populations. Indeed, Rab35 was strongly localized to sites of globular

581 actin that were also positive for filamentous actin (Fig.S8F).

582 Lastly, we used scanning electron microscopy to better visualize the remaining actin

583 network in ECs depleted of Rab35 or treated with CK-666. Qualitatively, there was reduced

584 filament density in the lamellipodia regions of the Rab35 depleted and CK-666 treated conditions

585 as compared with control (Fig. 6G). In Rab35 depleted ECs, we also observed elevated instances

586 of bundles of actin that were more disorganized in appearance as compared with control (Fig.

587 6G), potentially representing a compensatory effect for the lack of filamentous actin. Overall,

588 these results suggest that Rab35 is associated with regulating local sites of actin assembly.

590 Rab35 is required for blood vessel development in zebrafish. 
We next generated a Rab35 knockout in zebrafish using CRISPR/Cas9 gene editing to

592 test if Rab35 was also required for in vivo angiogenic processes [54]. In zebrafish, we targeted

593 both Rab35 paralogs, Rab35A and Rab35B. By sequence analysis we observed $100 \%$ indel

594 formation in $F_{0}$ injected zebrafish for both Rab35 paralogs (Fig. 7A). Double Rab35A/B knockout

595 was embryonic lethal marked by a lack of normal development as compared with scramble guide

596 injected controls, suggesting Rab35 is critical for normal embryonic development (Fig. 7B).

597 However, we did see a spectrum of developmental defects when the single-guide RNA amount

598 were diluted. In a vascular Lifeact-GFP expressing line injected with a sublethal dosage of

599 Rab35A/B single-guide RNA, we focused on actin defects. Here, we did not quantify vascular

600 defects due to the generalized tissue dysmorphogenesis of these embryos; alternatively, our goal

601 was to determine if similar actin accumulations occurred in vivo as observed in vitro. In line with

602 our in vitro data, we observed a significant increase in actin aggregations in the Rab35A/B

603 knockout group compared with controls (Fig. 7C,D). Similarly, overexpression of the DN Rab35

604 mutant or treatment with CK-666 promoted an increase in aberrant Rab35 accumulations,

605 presumably bound to actin (Fig. 7E,F). To subvert the lethality of global Rab35A/B deletion, we

606 generated chimeric embryos using blastomere transplants [55]. Transfer of Rab35A/B CRISPR

607 injected cells into a WT host generated mosaic intersomitic blood vessels (ISVs) allowing for

608 comparison of both WT and Rab35A/B null blood vessels side-by-side. Similar to in vitro results,

609 Rab35A/B null ISVs were dysmorphic, marked by a thin appearance and the absence of a lumen

610 as assessed by microangiography (Fig. 7G,H). Overall, these results indicate Rab35 is necessary

611 for organismal viability and actin homeostasis in vivo.

\section{DISCUSSION}

614 In the current work, we explored the contribution of Rab35 to angiogenic sprouting 615 behaviors vital to blood vessel development. The primary goal of this work was to interpret what 616 of the many reported functions of Rab35 matters most during blood vessel morphogenesis by 
617 systematically characterizing Rab35 itself and the downstream effector pathways. Using a 618 combination of 3D sprouting, biochemistry and in vivo gene editing, we demonstrate that Rab35's

619 most prominent function is to regulate actin dynamics during angiogenesis. More specifically, we 620 show that the GEF DENNd1c tethers active Rab35 to the actin cytoskeleton. Once localized to 621 actin, Rab35 promotes actin polymerization and remodeling required for sprout formation. 622 Additionally, we confirmed that Rab35 is required for blood vessel development in zebrafish. To 623 our knowledge, this is the first investigation demonstrating the requirement of Rab35 for blood 624 vessel function and the first investigation in any tissue dissecting Rab35's most dominant 625 biological role accounting for the most prominent effector pathways.

626 The genesis of the current project was originally aimed to characterize how podocalyxin 627 was trafficking in ECs, as this is still an outstanding question in the field. Our past work 628 demonstrated Rab27a, that was largely implicated in podocalyxin trafficking in epithelial cells, was 629 not related to this pathway [7], thus our very next candidate was Rab35. Others have 630 comprehensively established a direct association between Rab35 and podocalyxin as well as the 631 downstream impact on lumen biogenesis $[15,16]$. Our data in the current investigation once again 632 shows that endothelial trafficking signatures greatly differ from epithelial programs. More 633 specifically, we expansively tested for both localization and direct binding interaction between 634 Rab35 and podocalyxin of which we found none. However, this negative result prompted us to 635 further investigate Rab35 function during angiogenic sprouting.

636 Rab proteins are the most numerous subset of Ras family small guanosine 637 triphosphatases (GTPases). Rab proteins control biogenesis, movement, and docking of vesicles 638 in specific trafficking pathways by recruiting unique effector proteins to different membrane 639 compartments $[13,56]$. Rab35, in particular, has been shown to have many roles that vary by 640 tissue type, organism, and developmental stage. In distilling the literature, it can be argued that 641 Rab35 has four major effectors that mediate its function in vertebrates: RUSC, MICAL-L1, 642 ACAP2, and OCRL. Given Rab-family GTPases exert their function via effector interaction, we 
643 began by first establishing that Rab35 was required for sprouting, and then determined how each

644 effector contributed to the loss of Rab35 phenotype. Surprisingly, RUSC, MICAL-L1 and OCRL

645 either showed no phenotypic contribution to sprouting or failed to directly bind Rab35. The most

646 promising candidate ACAP2 exhibited the best phenotype for recapitulating the Rab35 loss of

647 function effect. In terms of ACAP2, we had several interesting findings. The predominant

648 hypothesis is that GTP Rab35 binds ACAP2 sequestering its ability to inactivate Arf6, resulting in

649 a gain of function for Arf6. In ECs, we could not confirm direct binding between ACAP2 and Arf6,

650 we also did not observe that Rab35 knockdown affected Arf6 activity as previously reported [17-

$65119,29,32,33,37,38]$. These previous reports were carried out in non-endothelial tissue, which

652 may explain the signaling discrepancy. However, we also overexpressed a CA Arf6, the predicted

653 outcome of loss of Rab35 in the aforementioned epithelial systems, and also could not phenocopy

654 the Rab35 loss of function effect on sprouting, again suggesting this signaling pathway is not

655 essential in ECs in the absence of Rab35.

A major finding was that the GEF DENNd1c played a key role in Rab35 function.

657 Canonically, GEFs primarily convert proteins from a GDP to GTP-bound state; however, 658 DENNd1c is evolutionarily divergent from both DENNd1a/b that solely control Rab35 GTPase 659 activity [41]. In our hands, loss of DENNd1c did not alter GTP activation, but controlled the 660 localization of Rab35 to actin fibrils. Knockdown of DENNd1c strongly phenocopied loss of Rab35 661 suggesting that localization to actin is a primary function of Rab35 during sprouting angiogenesis. Actin plays a pivotal role in angiogenesis both from a cell migration and vessel stabilization 663 aspect [57-62]. Loss of normal actin architecture has been shown to drastically affect virtually all 664 facets of blood vessel formation [63-67]. In this sense, our results are not surprising in that actin 665 misregulation promoted such a profound effect on sprouting parameters. However, given Rab35's

666 broad scope of function as well as never being characterized in angiogenic processes, it would 667 be exceedingly hard to predict. Moreover, actin regulation is typically known to be directly 668 controlled through more conventional signaling paradigms such as Rac1 and CDC42. Our results 
669 paint a novel scenario that trafficking-based regulators can control vital crosstalk with the actin

670 cytoskeleton. It is still an outstanding question what of the hundreds of cytoskeletal proteins

671 Rab35 is interfacing with to participate in actin regulation processes.

672 Overall, our investigation is the first to systematically rule out other known Rab35

673 pathways, highlighting Rab35's novel function in mediating actin dynamics during blood vessel

674 formation in vitro and in vivo. We believe this work is important not only from the vantage of

675 understanding EC biology and its unique trafficking signatures, but from a disease standpoint as

676 Rab35 is commonly upregulated in solid cancers [68]. In general, we contend that mapping

677 endothelial trafficking patterns will shed important light on how ECs orchestrate blood vessel

678 formation by integrating both cell-autonomous and collective-cell signaling.

ACKNOWLEDGEMENTS

681 Work was supported by funding from the National Heart Lung Blood Institute (Grant 682 1R56HL148450-01, R15HL156106-01A1, R01HL155921-01A1) (EJK).

\section{CONTRIBUTIONS}

685 CRF, HK and EJK performed all experiments. CRF and EJK wrote the manuscript. 
688

689

690

691

692

693

694

695

696

697

698

699

700

701

702

703

704

705

706

707

708

709

710

711

712

713

714

715

716

717

718

719

720

721

722

723

724

725

726

727

728

729

730

731

732

733

\section{LITERATURE CITED}

1. Kushner, E.J. and V.L. Bautch, Building blood vessels in development and disease. Curr Opin Hematol, 2013. 20(3): p. 231-6.

2. Chappell, J.C., D.M. Wiley, and V.L. Bautch, How blood vessel networks are made and measured. Cells Tissues Organs, 2012. 195(1-2): p. 94-107.

3. Ehling, M., et al., Notch controls retinal blood vessel maturation and quiescence. Development, 2013. 140(14): p. 3051-61.

4. Eilken, H.M. and R.H. Adams, Dynamics of endothelial cell behavior in sprouting angiogenesis. Curr Opin Cell Biol, 2010. 22(5): p. 617-25.

5. Kofler, N., et al., The Rab-effector protein RABEP2 regulates endosomal trafficking to mediate vascular endothelial growth factor receptor-2 (VEGFR2)dependent signaling. J Biol Chem, 2018. 293(13): p. 4805-4817.

6. Kempers, L., et al., The endosomal RIN2/Rab5C machinery prevents VEGFR2 degradation to control gene expression and tip cell identity during angiogenesis. Angiogenesis, 2021.

7. Francis, C.R., S. Claflin, and E.J. Kushner, Synaptotagmin-Like Protein 2a Regulates Angiogenic Lumen Formation via Weibel-Palade Body Apical Secretion of Angiopoietin-2. Arterioscler Thromb Vasc Biol, 2021: p. Atvbaha121316113.

8. Gross, S.J., et al., Notch regulates vascular collagen IV basement membrane through modulation of lysyl hydroxylase 3 trafficking. Angiogenesis, 2021.

9. Agola, J.O., et al., Rab GTPases as regulators of endocytosis, targets of disease and therapeutic opportunities. Clin Genet, 2011. 80(4): p. 305-18.

10. Jovic, M., et al., The early endosome: a busy sorting station for proteins at the crossroads. Histol Histopathol, 2010. 25(1): p. 99-112.

11. Kaksonen, M. and A. Roux, Mechanisms of clathrin-mediated endocytosis. Nat Rev Mol Cell Biol, 2018. 19(5): p. 313-326.

12. Hutagalung, A.H. and P.J. Novick, Role of Rab GTPases in membrane traffic and cell physiology. Physiol Rev, 2011. 91(1): p. 119-49.

13. Pfeffer, S., A model for Rab GTPase localization. Biochem Soc Trans, 2005. 33(Pt 4): p. 627-30.

14. Klinkert, K. and A. Echard, Rab35 GTPase: A Central Regulator of Phosphoinositides and F-actin in Endocytic Recycling and Beyond. Traffic, 2016. 17(10): p. 1063-77.

15. Mrozowska, P.S. and M. Fukuda, Regulation of podocalyxin trafficking by Rab small GTPases in 2D and 3D epithelial cell cultures. J Cell Biol, 2016. 213(3): p. 355-69.

16. Klinkert, K., et al., Rab35 GTPase couples cell division with initiation of epithelial apico-basal polarity and lumen opening. Nature Communications, 2016. 7(1): p. 11166.

17. Kobayashi, H. and M. Fukuda, Rab35 regulates Arf6 activity through centaurin$\beta 2$ (ACAP2) during neurite outgrowth. J Cell Sci, 2012. 125(Pt 9): p. 2235-43.

18. Miyamoto, Y., et al., Rab35, acting through ACAP2 switching off Arf6, negatively regulates oligodendrocyte differentiation and myelination. Mol Biol Cell, 2014. 25(9): p. 1532-42. 
19. Bhat, S., et al., Rab35 and its effectors promote formation of tunneling nanotubes in neuronal cells. Scientific Reports, 2020. 10(1): p. 16803.

20. Rahajeng, J., et al., MICAL-L1 is a tubular endosomal membrane hub that connects Rab35 and Arf6 with Rab8a. Traffic, 2012. 13(1): p. 82-93.

21. Frémont, S., et al., Oxidation of F-actin controls the terminal steps of cytokinesis. Nature Communications, 2017. 8(1): p. 14528.

22. Zhang, J., et al., Rab35 controls actin bundling by recruiting fascin as an effector protein. Science, 2009. 325(5945): p. 1250-4.

23. Remsburg, C., M. Testa, and J.L. Song, Rab35 regulates skeletogenesis and gastrulation by facilitating actin remodeling and vesicular trafficking. Cells Dev, 2021. 165.

24. Campeau, E., et al., A versatile viral system for expression and depletion of proteins in mammalian cells. PLoS One, 2009. 4(8): p. e6529.

25. Nakatsu, M.N., J. Davis, and C.C. Hughes, Optimized fibrin gel bead assay for the study of angiogenesis. J Vis Exp., 2007(3): p. 186. doi: 10.3791/186. Epub 2007 Apr 29.

26. Nakatsu, M.N. and C.C. Hughes, An optimized three-dimensional in vitro model for the analysis of angiogenesis. Methods Enzymol., 2008. 443:65-82.(doi): p. 10.1016/S0076-6879(08)02004-1.

27. Kushner, E.J., et al., Excess centrosomes disrupt endothelial cell migration via centrosome scattering. J Cell Biol, 2014. 206(2): p. 257-72.

28. Francis, C.R. and E.J. Kushner, Capturing membrane trafficking events during 3D angiogenic development in vitro. Microcirculation, 2021: p. e12726.

29. Chesneau, L., et al., An ARF6/Rab35 GTPase cascade for endocytic recycling and successful cytokinesis. Curr Biol, 2012. 22(2): p. 147-53.

30. Dambournet, D., et al., Rab35 GTPase and OCRL phosphatase remodel lipids and F-actin for successful cytokinesis. Nature Cell Biology, 2011. 13(8): p. 981988.

31. Kouranti, I., et al., Rab35 regulates an endocytic recycling pathway essential for the terminal steps of cytokinesis. Curr Biol, 2006. 16(17): p. 1719-25.

32. Biesemann, A., et al., Rab35 protein regulates evoked exocytosis of endothelial Weibel-Palade bodies. J Biol Chem, 2017. 292(28): p. 11631-11640.

33. Jackson, T.R., et al., ACAPs are arf6 GTPase-activating proteins that function in the cell periphery. J Cell Biol, 2000. 151(3): p. 627-38.

34. Cauvin, C., et al., Rab35 GTPase Triggers Switch-like Recruitment of the Lowe Syndrome Lipid Phosphatase OCRL on Newborn Endosomes. Curr Biol, 2016. 26(1): p. 120-8.

35. Lin, L., et al., Rab35/ACAP2 and Rab35/RUSC2 Complex Structures Reveal Molecular Basis for Effector Recognition by Rab35 GTPase. Structure, 2019. 27(5): p. 729-740.e3.

36. Powelka, A.M., et al., Stimulation-dependent recycling of integrin beta1 regulated by ARF6 and Rab11. Traffic, 2004. 5(1): p. 20-36.

37. Boshans, R.L., et al., ADP-ribosylation factor 6 regulates actin cytoskeleton remodeling in coordination with Rac1 and RhoA. Mol Cell Biol, 2000. 20(10): p. 3685-94. 
38. Al-Awar, O., et al., Separation of membrane trafficking and actin remodeling functions of ARF6 with an effector domain mutant. Mol Cell Biol, 2000. 20(16): p. 5998-6007.

39. Parachoniak, C.A., et al., GGA3 functions as a switch to promote Met receptor recycling, essential for sustained ERK and cell migration. Dev Cell, 2011. 20(6): p. 751-63.

40. Marat, A.L. and P.S. McPherson, The connecdenn family, Rab35 guanine nucleotide exchange factors interfacing with the clathrin machinery. J Biol Chem, 2010. 285(14): p. 10627-37.

41. Marat, A.L., M.S. loannou, and P.S. McPherson, Connecdenn 3/DENND1C binds actin linking Rab35 activation to the actin cytoskeleton. Mol Biol Cell, 2012. 23(1): p. 163-75.

42. Chaineau, M., M.S. Ioannou, and P.S. McPherson, Rab35: GEFs, GAPs and effectors. Traffic, 2013. 14(11): p. 1109-17.

43. Allaire, P.D., et al., The Connecdenn DENN domain: a GEF for Rab35 mediating cargo-specific exit from early endosomes. Mol Cell, 2010. 37(3): p. 370-82.

44. Mullins, R.D., J.A. Heuser, and T.D. Pollard, The interaction of Arp2/3 complex with actin: nucleation, high affinity pointed end capping, and formation of branching networks of filaments. Proc Natl Acad Sci U S A, 1998. 95(11): p. 6181-6.

45. Mullins, R.D., J.A. Heuser, and T.D. Pollard, The interaction of Arp2/3 complex with actin: Nucleation, high affinity pointed end capping, and formation of branching networks of filaments. Proceedings of the National Academy of Sciences, 1998. 95(11): p. 6181-6186.

46. Hetrick, B., et al., Small molecules CK-666 and CK-869 inhibit actin-related protein $2 / 3$ complex by blocking an activating conformational change. Chem Biol, 2013. 20(5): p. 701-12.

47. Farrants, H., et al., Chemogenetic Control of Nanobodies. Nat Methods, 2020. 17(3): p. 279-282.

48. Morozova, K.S., et al., Far-red fluorescent protein excitable with red lasers for flow cytometry and superresolution STED nanoscopy. Biophys J, 2010. 99(2): p. L13-5.

49. Lauffenburger, D.A. and A.F. Horwitz, Cell migration: a physically integrated molecular process. Cell, 1996. 84(3): p. 359-69.

50. Svitkina, T.M. and G.G. Borisy, Arp2/3 complex and actin depolymerizing factor/cofilin in dendritic organization and treadmilling of actin filament array in lamellipodia. J Cell Biol, 1999. 145(5): p. 1009-26.

51. Barry, D.J., et al., Open source software for quantification of cell migration, protrusions, and fluorescence intensities. J Cell Biol, 2015. 209(1): p. 163-80.

52. Milligan, R.A., M. Whittaker, and D. Safer, Molecular structure of F-actin and location of surface binding sites. Nature, 1990. 348(6298): p. 217-221.

53. Lee, Chi W., et al., Dynamic Localization of G-Actin during Membrane Protrusion in Neuronal Motility. Current Biology, 2013. 23(12): p. 1046-1056.

54. Hoshijima, K., et al., Highly Efficient CRISPR-Cas9-Based Methods for Generating Deletion Mutations and FO Embryos that Lack Gene Function in Zebrafish. Dev Cell, 2019. 51(5): p. 645-657.e4. 
825

55. White, R.M., et al., Transparent adult zebrafish as a tool for in vivo transplantation analysis. Cell Stem Cell, 2008. 2(2): p. 183-9.

56. Pfeffer, S. and D. Aivazian, Targeting Rab GTPases to distinct membrane compartments. Nat Rev Mol Cell Biol, 2004. 5(11): p. 886-96.

57. Gebala, V., et al., Blood flow drives lumen formation by inverse membrane blebbing during angiogenesis in vivo. Nat Cell Biol, 2016. 18(4): p. 443-50.

58. Phng, L.K., et al., Formin-mediated actin polymerization at endothelial junctions is required for vessel lumen formation and stabilization. Dev Cell, 2015. 32(1): p. 123-32.

59. Paatero, I., et al., Junction-based lamellipodia drive endothelial cell rearrangements in vivo via a VE-cadherin-F-actin based oscillatory cell-cell interaction. Nature Communications, 2018. 9(1): p. 3545.

60. van Geemen, D., et al., F-actin-anchored focal adhesions distinguish endothelial phenotypes of human arteries and veins. Arterioscler Thromb Vasc Biol, 2014. 34(9): p. 2059-67.

61. Norden, P.R., Z. Sun, and G.E. Davis, Control of endothelial tubulogenesis by Rab and Ral GTPases, and apical targeting of caveolin-1-labeled vacuoles. PLoS One, 2020. 15(6): p. e0235116.

62. Iruela-Arispe, M.L. and G.E. Davis, Cellular and molecular mechanisms of vascular lumen formation. Dev Cell, 2009. 16(2): p. 222-31.

63. Bayless, K.J. and G.A. Johnson, Role of the cytoskeleton in formation and maintenance of angiogenic sprouts. J Vasc Res, 2011. 48(5): p. 369-85.

64. Barry, D.M., et al., Rasip1-Mediated Rho GTPase Signaling Regulates Blood Vessel Tubulogenesis via Nonmuscle Myosin II. Circ Res, 2016. 119(7): p. 81026.

65. Barry, D.M., et al., Cdc42 is required for cytoskeletal support of endothelial cell adhesion during blood vessel formation in mice. Development, 2015. 142(17): p. 3058-70.

66. Kondrychyn, I., et al., Marcks/1 modulates endothelial cell mechanoresponse to haemodynamic forces to control blood vessel shape and size. Nat Commun, 2020. 11(1): p. 5476.

67. Norden, P.R., et al., Cdc42 and k-Ras Control Endothelial Tubulogenesis through Apical Membrane and Cytoskeletal Polarization: Novel Stimulatory Roles for GTPase Effectors, the Small GTPases, Rac2 and Rap1b, and Inhibitory Influence of Arhgap31 and Rasa1. PLoS One, 2016. 11(1): p. e0147758.

68. Villagomez, F.R., et al., The role of the oncogenic Rab35 in cancer invasion, metastasis, and immune evasion, especially in leukemia. Small GTPases, 2018: p. 1-12.

69. Gibson, D.G., et al., Enzymatic assembly of DNA molecules up to several hundred kilobases. Nat Methods, 2009. 6(5): p. 343-5.

70. Webb, A.M., et al., EHD2 modulates DIl4 endocytosis during blood vessel development. Microcirculation, 2021: p. e12740.

71. Li, P., R.M. White, and L.I. Zon, Transplantation in zebrafish. Methods Cell Biol, 2011. 105: p. 403-17.

72. Kawakami, K., Tol2: a versatile gene transfer vector in vertebrates. Genome Biol, 2007. 8 Suppl 1(Suppl 1): p. S7. 
871 73. Suster, M.L., et al., Transgenesis in zebrafish with the to/2 transposon system.

872 Methods Mol Biol., 2009. 561:41-63.(doi): p. 10.1007/978-1-60327-019-9_3.

873 74. Balciunas, D., et al., Harnessing a high cargo-capacity transposon for genetic

$874 \quad$ applications in vertebrates. PLoS Genet, 2006. 2(11): p. e169.

875 75. Watanabe, K., et al., Networks of polarized actin filaments in the axon initial segment provide a mechanism for sorting axonal and dendritic proteins. Cell Rep, 2012. 2(6): p. 1546-53. 

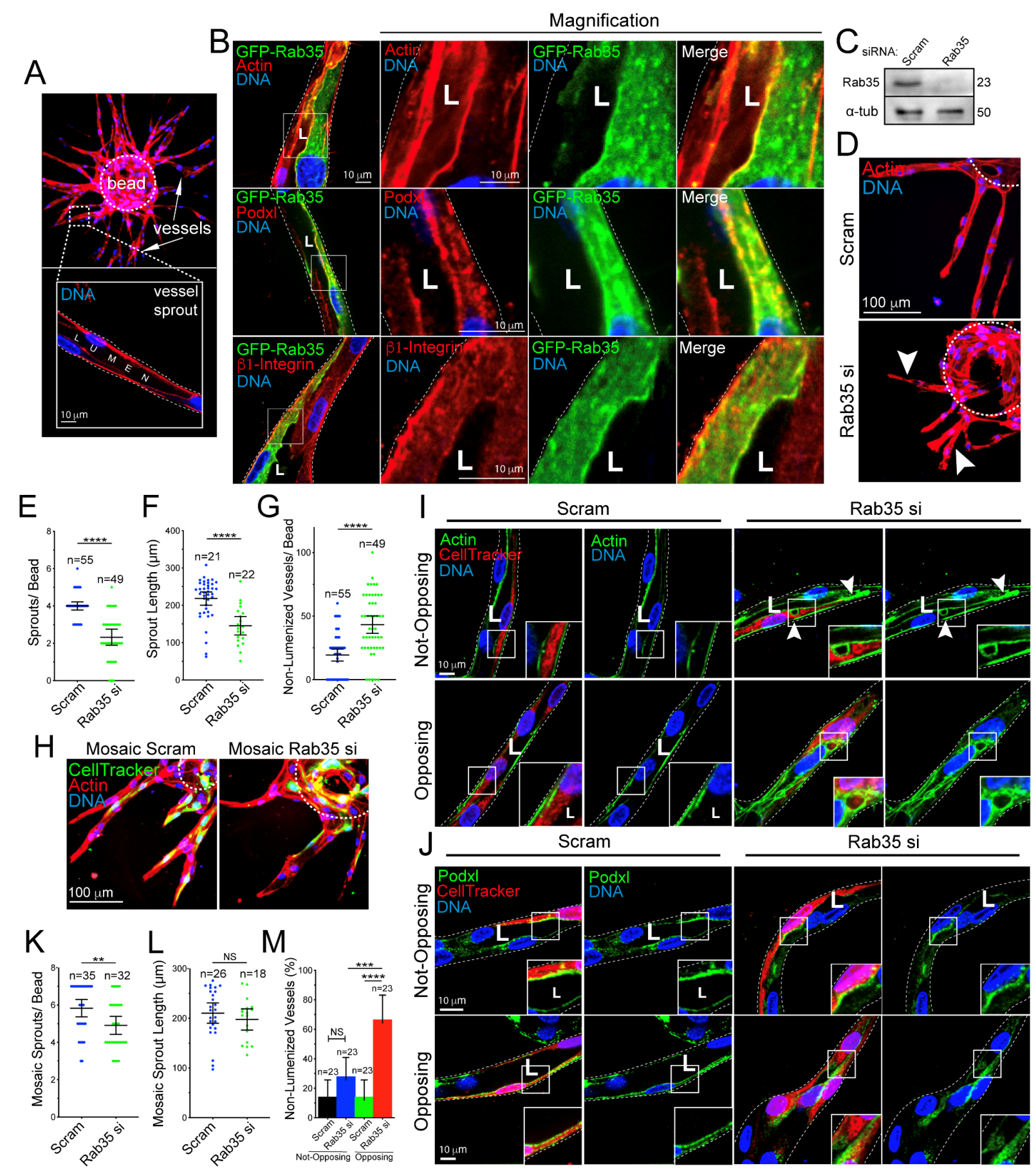

883

884

885

886

887

888

889

890

Figure 1. Rab35 is an apical membrane protein required for sprout formation. (A) Representative images of the fibrin-bead assay (FBA) at low and high magnification. Arrows mark sprout structures. Inset depicts lumenized sprout. (B) GFP-Rab35 localization in endothelial sprouts with actin (top panels), podocalyxin (Podxl, middle panels), and $\beta 1$-integrin (bottom panels). (C) Western blot confirmation of siRNA knockdown of Rab35. (D) Representative image of scramble (Scram) control and Rab35 siRNA (si) knock-down (KD) sprouts. Arrowheads denote 
891 short and non-lumenized sprouts. Dashed lines outline the microbead. (E-G) Graphs of indicated

892 sprouting parameters between groups. (H) Representative images of sprout morphology of

893 mosaic Scram and Rab35 KD cells, green indicates cell tracker of siRNA treated cells. $(\mathbf{I}, \mathbf{J})$

894 Representative images of non-opposing (top panels, an isolated siRNA treated cell) and opposing

895 (bottom panels, two adjacent siRNA treated cells) cells stained as indicated. Arrowheads denote

896 aberrant actin accumulations (K-M) Quantification of indicated parameters across groups. In all

897 images $L$ denotes lumen. ${ }^{* *} p<0.01,{ }^{* * *} p<0.001,{ }^{* * *} p<0.0001$, NS=Non-Significant. Error bars

898 represent $95 \%$ confidence intervals. $\mathrm{N}=$ number of sprouts. Insets are areas of higher

899 magnification. White dotted lines mark sprout exterior. All experiments were done using Human

900 umbilical vein endothelial cells in triplicate. 

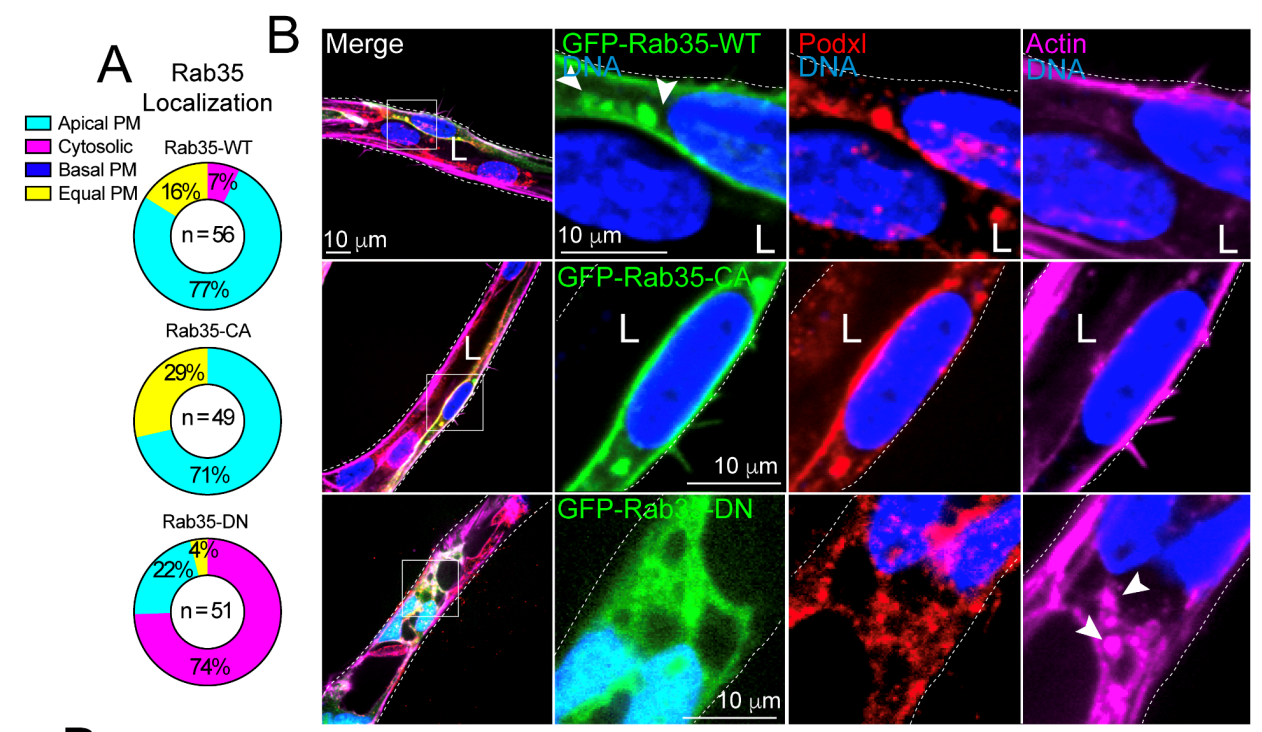

C

GFP-Rab35 Rescue

Lumen Formation

Non-Lumenized

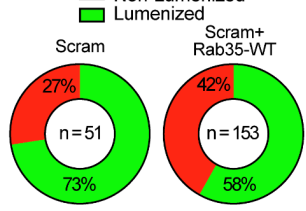

Rab35 si

Rab35 si+
Rab35-WT

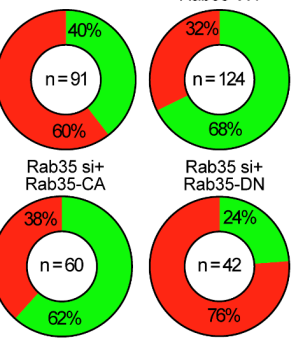

$\mathrm{D}_{+}$GFP-Cytosolic

Scram
Rab35 si
FP-Rab35-WT

Rab35 si

Rab35 si

Rab35 si

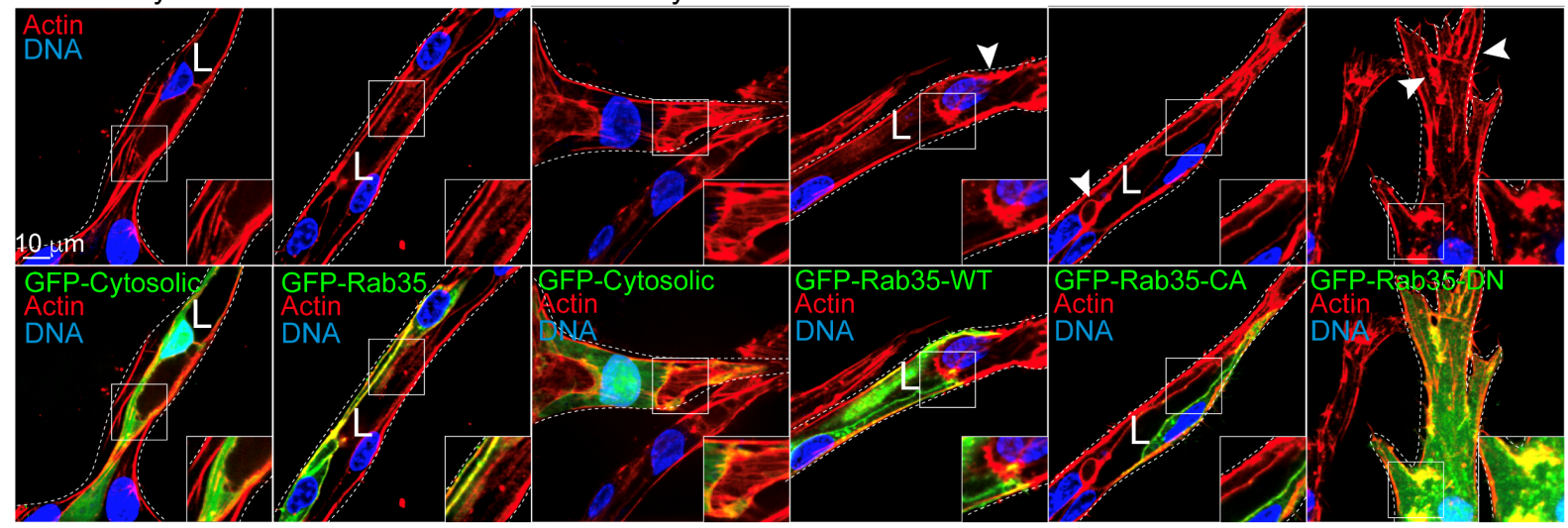

942

943

944

945

946

947

948

949

950

951

952

953

954

955

956

957

958

959

960
Figure 2. Rab35 mutant localization and rescue in endothelial sprouts. (A) Quantification of GFP-Rab35 wild-type (WT), constitutively active (CA), and dominant-negative (DN) localization in endothelial sprouts. Apical plasma membrane (PM, uniformly localized to apical membrane), basal PM (Rab35 uniformly located at the basal membrane), cytosolic (localized in the cytoplasm), equal PM (Rab35 equally distributed between the apical and basal membranes). $\mathrm{N}=$ number of cells. (B) GFP-Rab35 WT (top panels), CA (middle panels), and DN (bottom panels) localization in endothelial sprouts. Co-staining with Podocalyxin (Podxl) and actin. Arrowheads in top panels denote Rab35 apical localization and puncta. Arrowheads in bottom panels denote abnormal accumulations of actin. (C) Quantification of lumen formation in described conditions. N=number of sprouts. (D) Representative images of Rab35 KD sprouts rescued with either GFP-cytosolic, (control), or GFP-Rab35-WT/CA/DN. Arrowheads denote actin accumulations. White dotted lines mark sprout exterior. L denotes lumen in all images. Insets are areas of higher magnification. All experiments were done using Human umbilical vein endothelial cells in triplicate. 

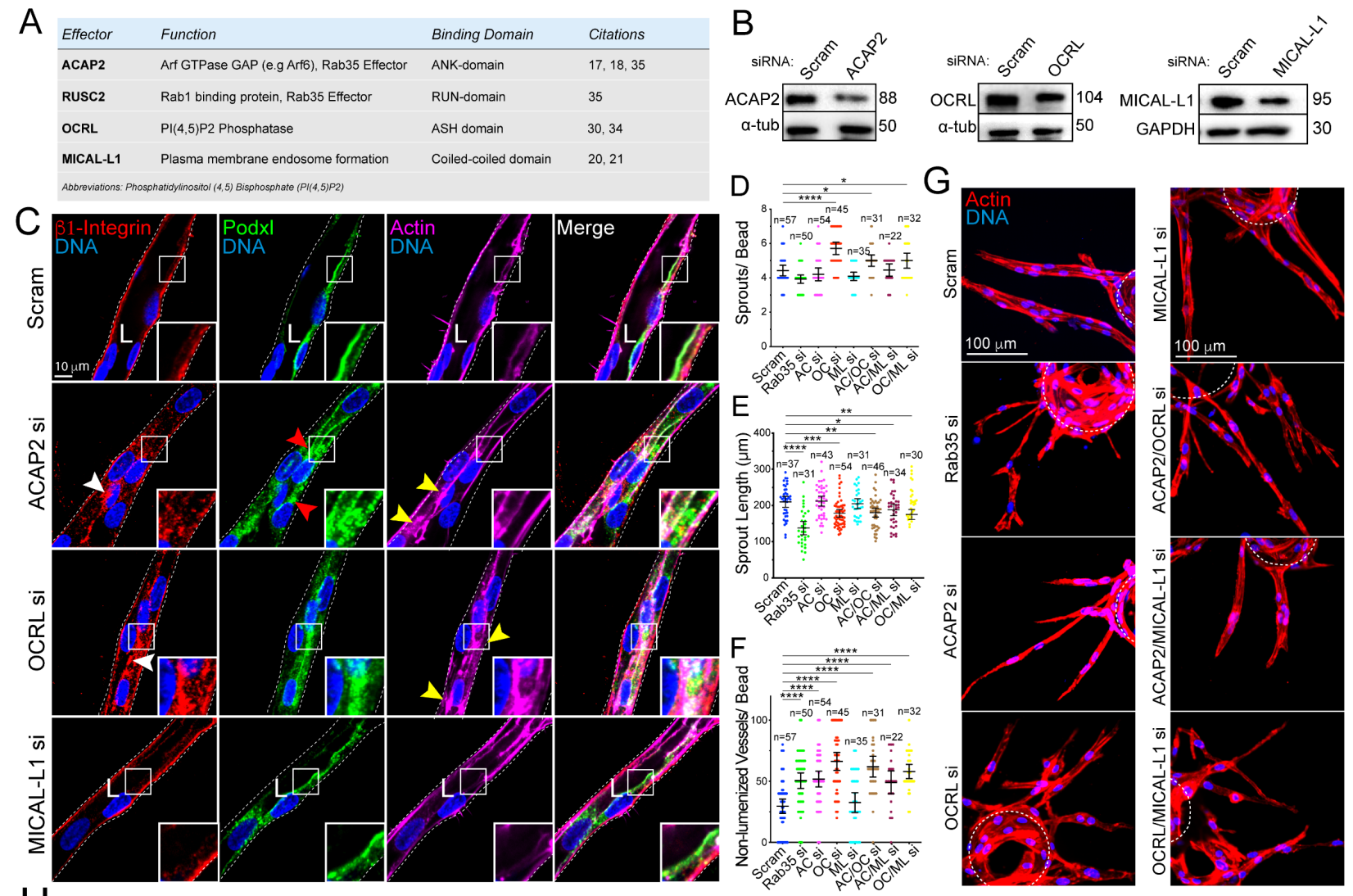

$\mathrm{H}$
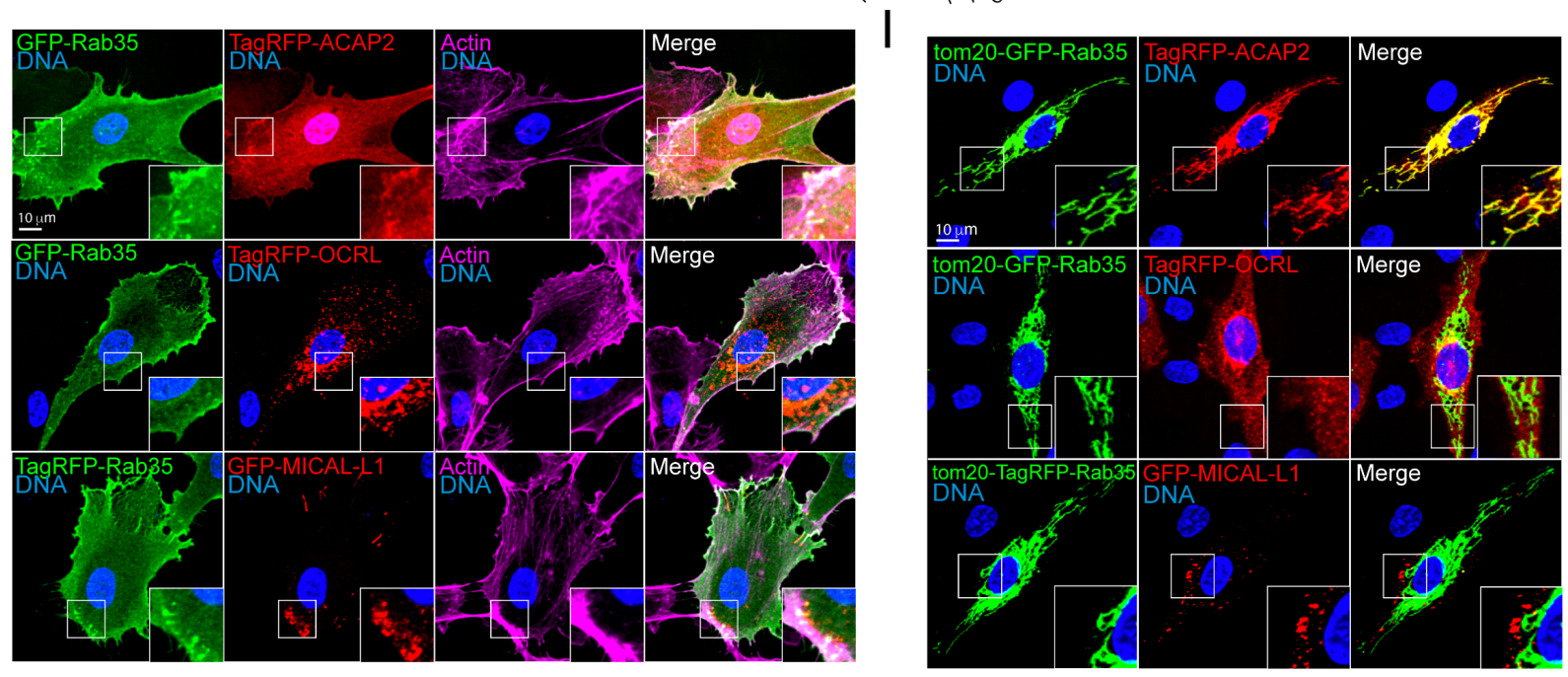

961

962

963

964

965

966

967

968

969

970
Figure 3. Rab35 effector localization and necessity to endothelial sprout formation. (A) Table listing each effector, respective function, and citations. (B) ACAP2, OCRL, and MICAL-L1 knockdown (KD) validation by western blotting. (C) Representative images of siRNA (si)-mediated KD of each effector. White arrowhead denotes abnormal localization of $\beta 1$-integrin. Red arrowheads denote abnormal podocalyxin (Podxl) localization. Yellow arrowheads denote abnormal actin accumulations. White dotted lines mark sprout exterior. (D-F) Graphs of indicated sprout parameters between groups. ACAP2 (AC), OCRL (OC), and MICAL-L1 (ML). N= number of sprouts. (G) Representative images of sprout morphology between indicated groups. Dashed 
971 lines outline microbeads. (H) Two-dimensional localization of GFP-Rab35 or TagRFP-Rab35 with

972 indicated effectors and stained for actin. (I) Representative images of mitochondrial mis-

973 localization experiment. Rab35 was unnaturally tethered to the mitochondria with a tom20 N-

974 terminal tag to test if indicated effectors were also mislocalized to the mitochondria. In all images

$975 \mathrm{~L}$ denotes lumen. ${ }^{*} p<0.05,{ }^{* *} p<0.01,{ }^{* * *} p<0.001,{ }^{* * *} p<0.0001, \mathrm{NS}=$ Non-Significant. Error

976 bars represent $95 \%$ confidence intervals. $\mathrm{N}=$ number of sprouts. Insets are areas of higher

977 magnification. All experiments were done using Human umbilical vein endothelial cells in

978 triplicate. 

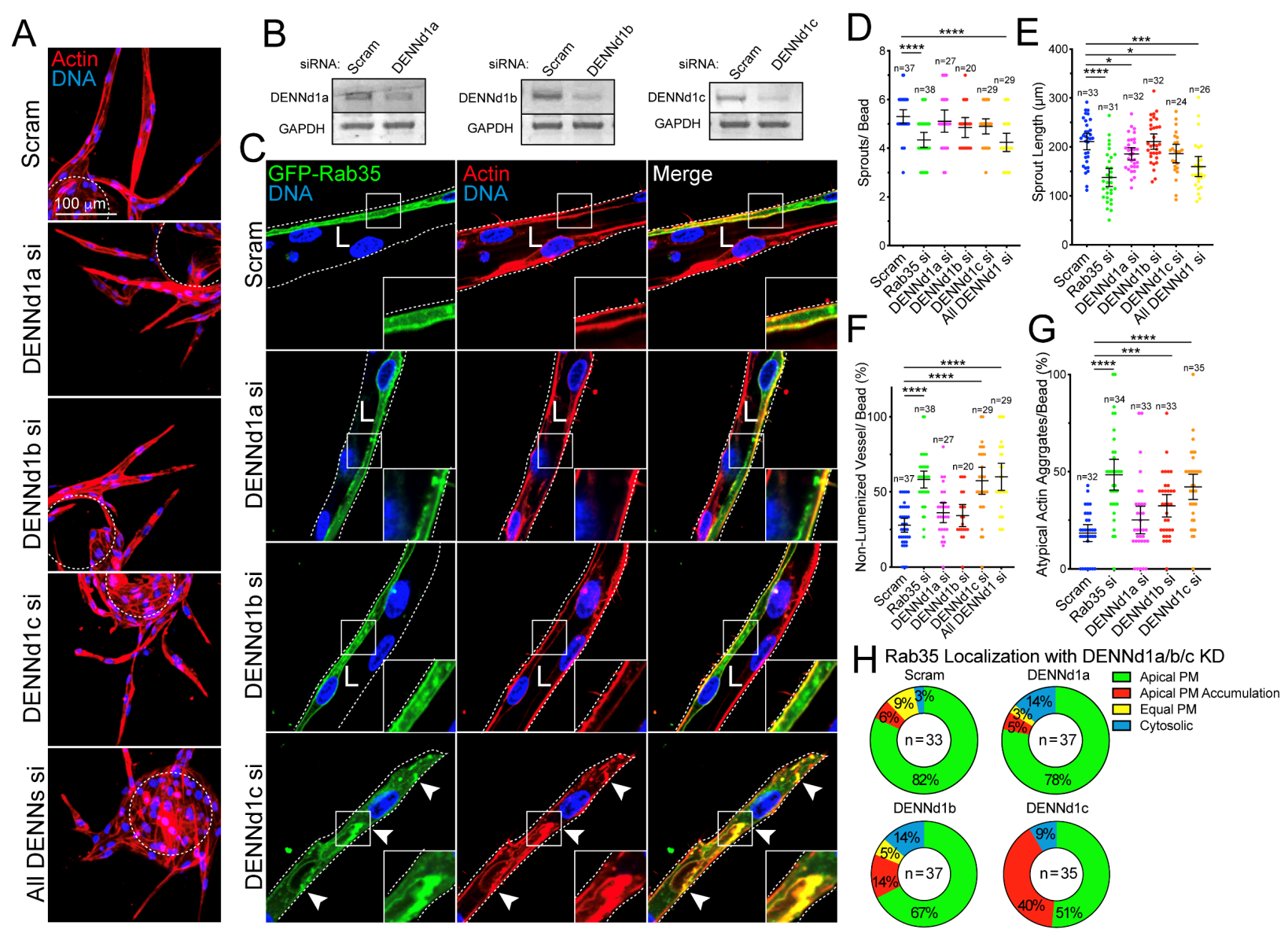

Figure 4. DENNd1c is required for sprouting and Rab35 function. (A) Sprout morphology of scramble (Scram), DENNd1a-c and combined siRNA (si)-treated sprouts, stained with actin to denote the general morphology. Dashed line denotes microbead. (B) Knockdown confirmations for DENNd1a-c by RT-PCR. (C) Representative images of siRNA knockdowns described in A with GFP-Rab35 localization. $L$ denotes lumen and arrowheads denote abnormal actin accumulations. White dotted lines mark sprout exterior. (D-G) Graphs of indicated sprout parameters across groups. $\mathrm{N}=$ number of sprouts. (H) GFP-Rab35 localization in DENNd1a-c siRNA-treated sprouts. Localizations were binned to apical plasma membrane (PM, Rab35 $>80 \%$ at apical membrane), apical PM accumulations (non-continuous, visible puncta), equal PM (equally enriched at apical and basal membranes), and cytosolic. $\mathrm{N}=$ number of sprouts. ${ }^{*} \mathrm{p}<$ $0.05,{ }^{* * *} p<0.001,{ }^{* * *} p<0.0001$, NS=Non-Significant. Error bars represent $95 \%$ confidence intervals. Insets are areas of higher magnification. All experiments were done using Human

1036

1037 umbilical vein endothelial cells in triplicate.

1038

1039 

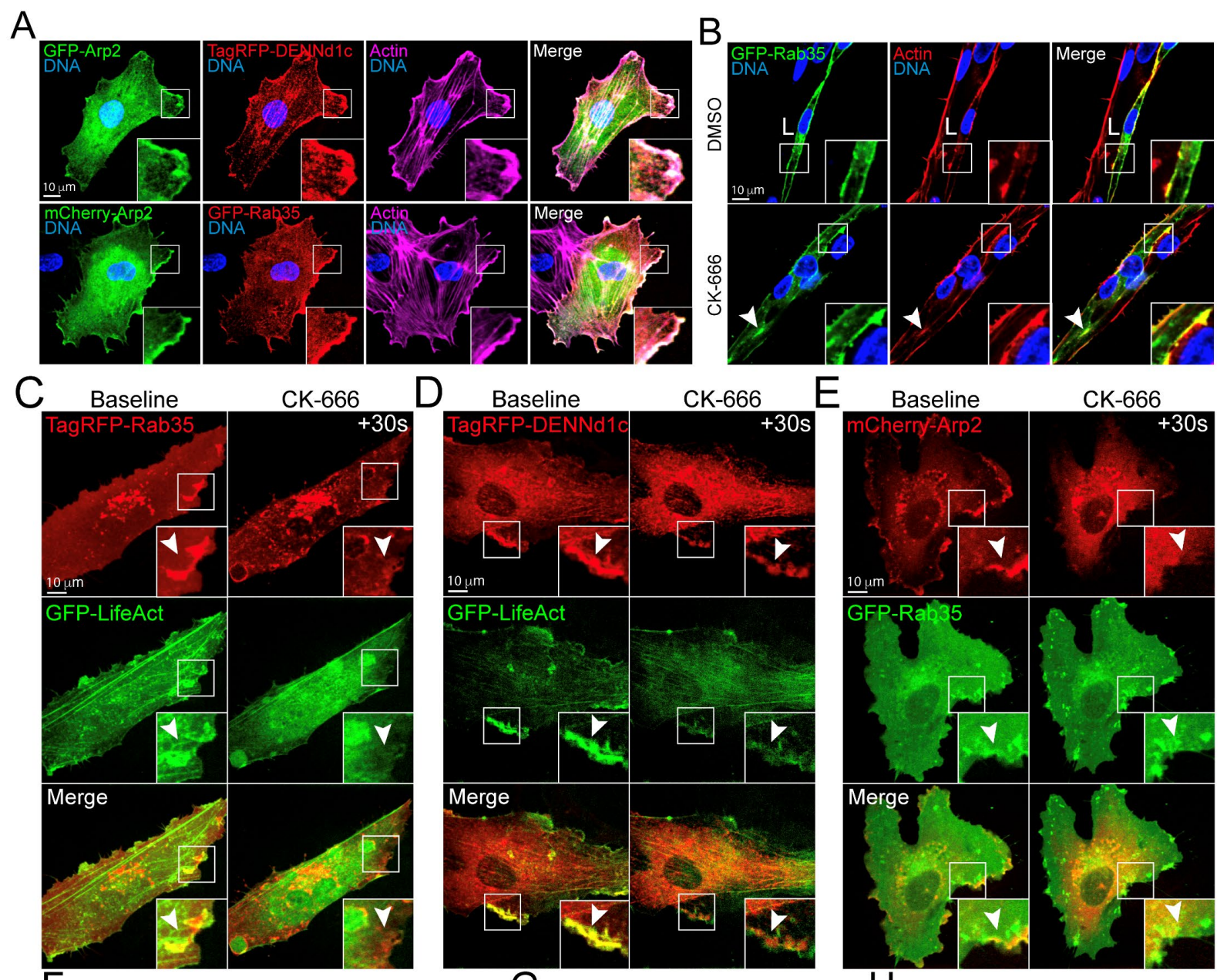

F Mitochondria Mis-localization and Release

Baseline

CK-666

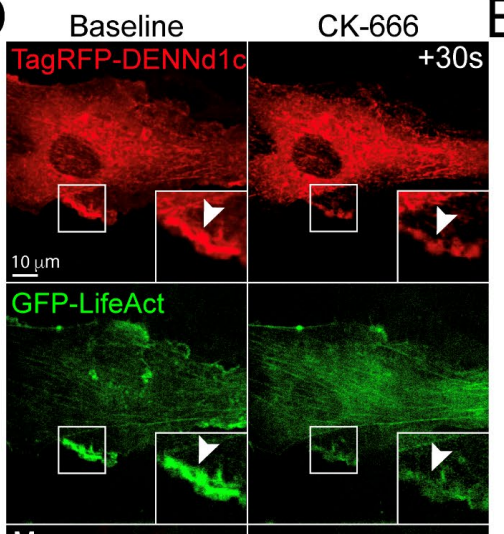

E Baseline

CK-666

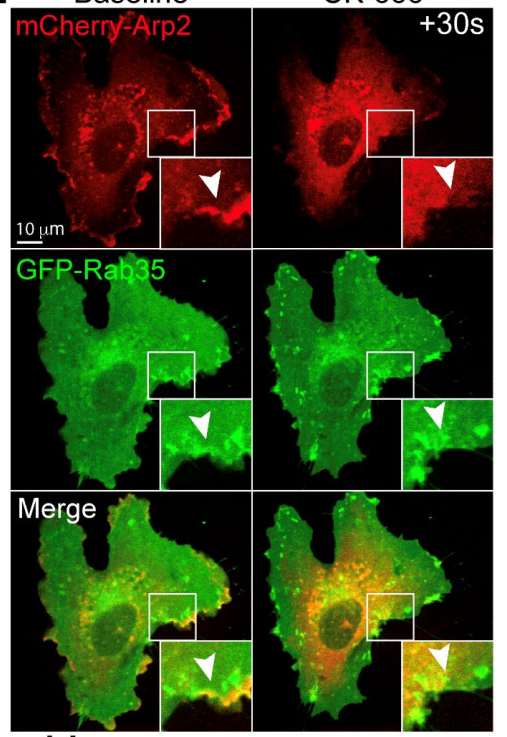

$\mathrm{H}$

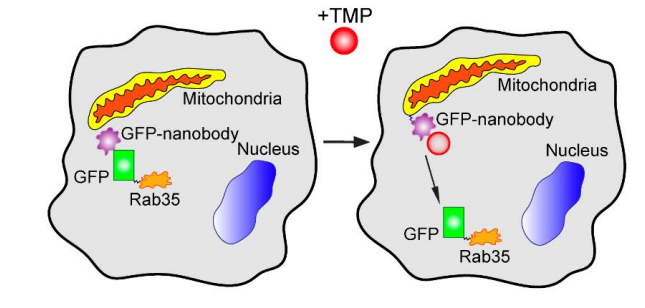

G
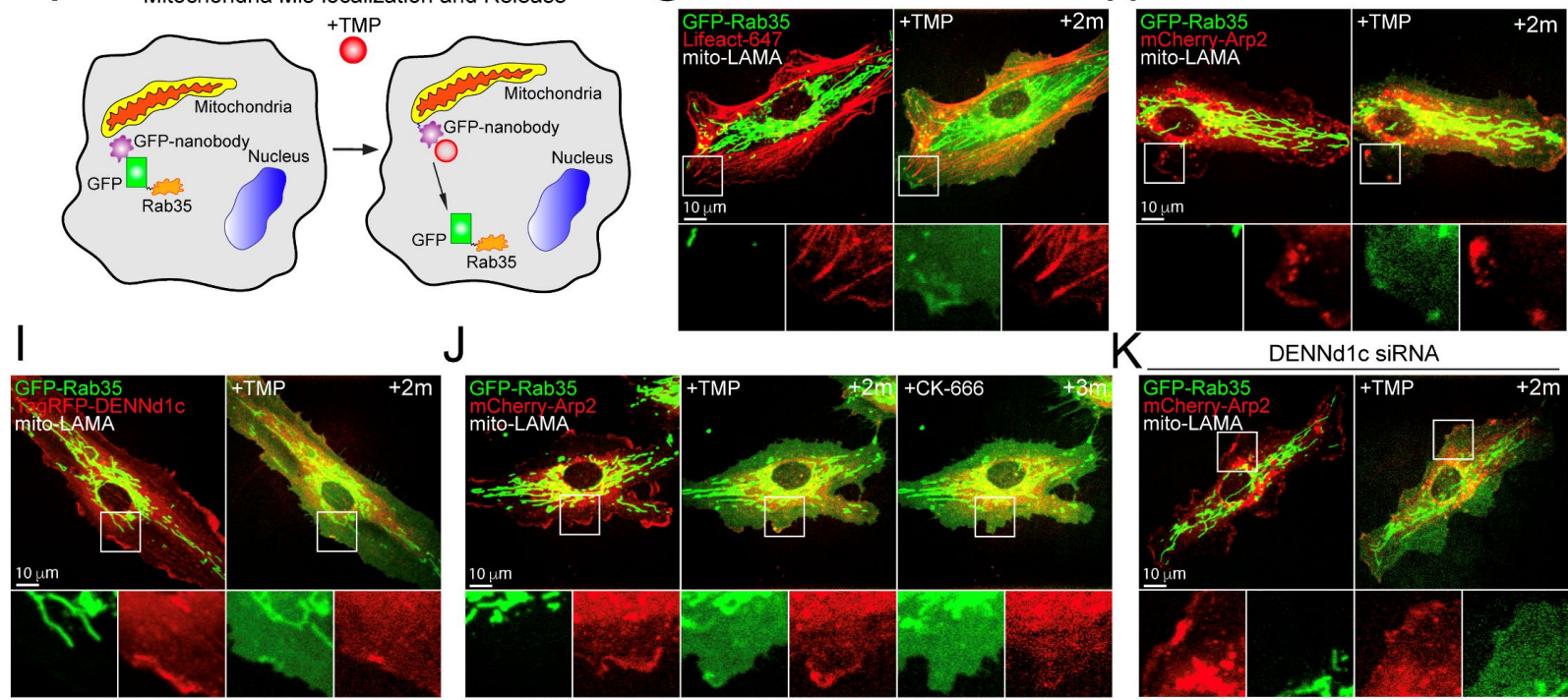

$\mathrm{J}$
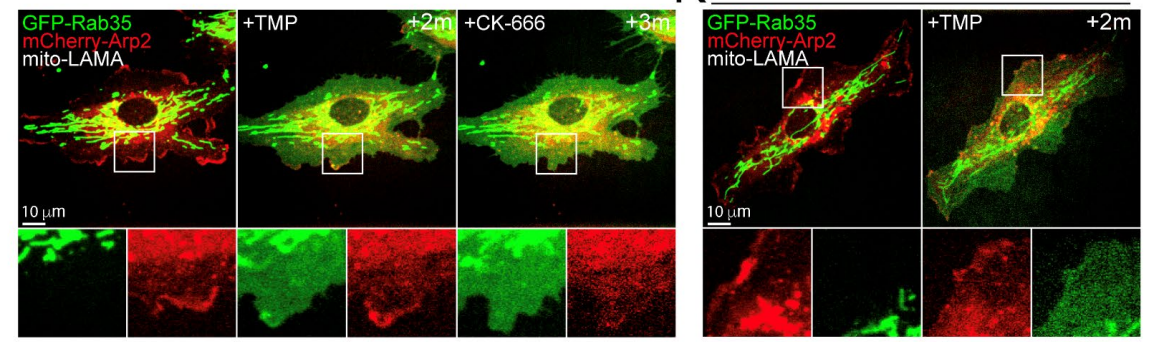

Figure 5. Rab35 localizes to cortical actin. (A) Two-dimensional localization of GFP-Arp2 with DENNd1c (top panels) and GFP-Rab35 (bottom panels). (B) Representative images of DMSO and CK-666 (Arp Inhibitor) treated sprouts expressing GFP-Rab35. L denotes lumen. (C,D) Live 
1051

1052

1053

1054

1055

1056

1057

1058

1059

1060

1061

1062

1063

1064

1065

1066

1067

1068

1069

1070

1071

1072

1073

1074

1075

1076

1077

1078

1079

1080

1081

1082

1083

1084

1085

1086

1087

1088

1089

1090

1091

1092

1093

1094

1095

1096

1097

1098

1099

1100

1101 imaging of GFP-Rab35 or tagRFP-DENNd1c with TagRFP647-LifeAct at baseline and after treatment with CK-666. White arrowheads denote disappearance of Rab35 puncta over time. (E) Representative live-images of a cell expressing mCherry-Arp2 and GFP-Rab35 before and after CK-666 treatment White arrowheads denote disappearance of Rab35 puncta over time. (F) Cartoon of a mitochondria-localized GFP-nanobody and controlled release of GFP-Rab35 upon treatment with Trimethoprim (TMP). In the absence of TMP the nanobody sequesters GFP or GFP-tagged proteins. In the presence of TMP the GFP cargo is released. (G) Live-image of a cell expressing GFP-Rab35, TagRFP647 (647)-LifeAct and ligand-modulated antibody fragments targeted to the mitochondria (mito-LAMA) before and after TMP administration. (H) Live-image of a cell expressing GFP-Rab35, mCherry-Arp2 and mito-LAMA before and after TMP administration. (I) Live-image of a cell expressing GFP-Rab35, TagRFP-DENNd1c and mitoLAMA before and after TMP administration. (J) Live-image of a cell expressing GFP-Rab35, mCherry-Arp2 and mito-LAMA before and after TMP administration and then treated with CK666. (K) Live-image of a cell expressing GFP-Rab35, mCherry-Arp2 and mito-LAMA treated with DENNd1c siRNA (si) before and after TMP administration. Insets are areas of higher magnification. All experiments were done using Human umbilical vein endothelial cells in triplicate. 

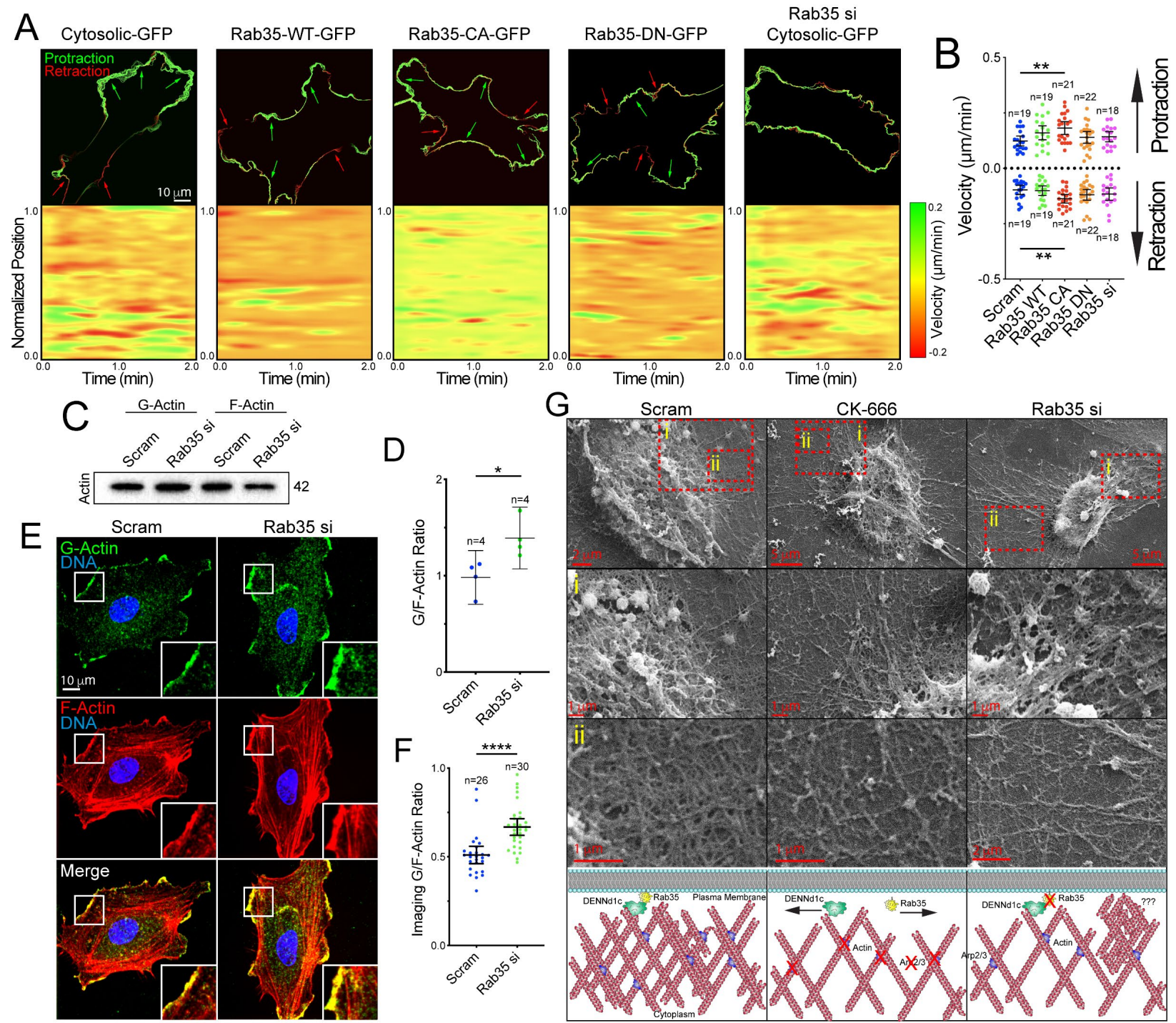

Figure 6. Rab35 regulates actin dynamics. (A) Top panels depict change in membrane velocities over time in described conditions. Green represents protraction and red represents retraction of the membrane. Arrows indicate directionality. The bottom panels are heat maps in which the Red is indicative of retractive movement and green is protractive movement over time. Yellow indicates no change in velocity. (B) Quantification of cell membrane velocities between indicated groups. Above the dashed line is the protractive velocities and below the dashed line is retractive velocities. $\mathrm{N}=$ number of cells. (C) Western blot of globular and filamentous actin in siRNA (si)-treated groups. (D) Quantification of the ratio of globular to filamentous actin from blots represented in panel C. (E) Representative images of cells stained for globular and filamentous actin between indicated conditions. (F) Quantification of the ratio of globular to filamentous actin fluorescent intensities. (G) Scanning electron microscopy of filament network between groups. Top panel is the lowest magnification with higher magnifications in panels (i) and (ii). Bottomcartoon representation of SEM filament network and hypothesized role of Rab35. * $p<0.05$, ** $\mathrm{p}<0.01,{ }^{* * * *} \mathrm{p}<0.0001, \mathrm{NS}=$ Non-Significant. Error bars represent $95 \%$ confidence intervals. 
1118 Insets are areas of higher magnification. All experiments were done using Human umbilical vein 1119 endothelial cells in triplicate.

1120

1121

1122

1123

1124

1125

1126

1127

1128

1129

1130

1131

1132

1133

1134

1135

1136

1137

1138

1139

1140

1141

1142

1143

1144

1145

1146

1147

1148

1149

1150

1151

1152

1153

1154

1155

1156

1157

1158

1159

1160

1161

1162

1163

1164

1165

1166

1167

1168 
bioRxiv preprint doi: https://doi.org/10.1101/2022.01.13.476231; this version posted January 14, 2022. The copyright holder for this preprint (which was not certified by peer review) is the author/funder, who has granted bioRxiv a license to display the preprint in perpetuity. It is made available under aCC-BY-NC 4.0 International license.

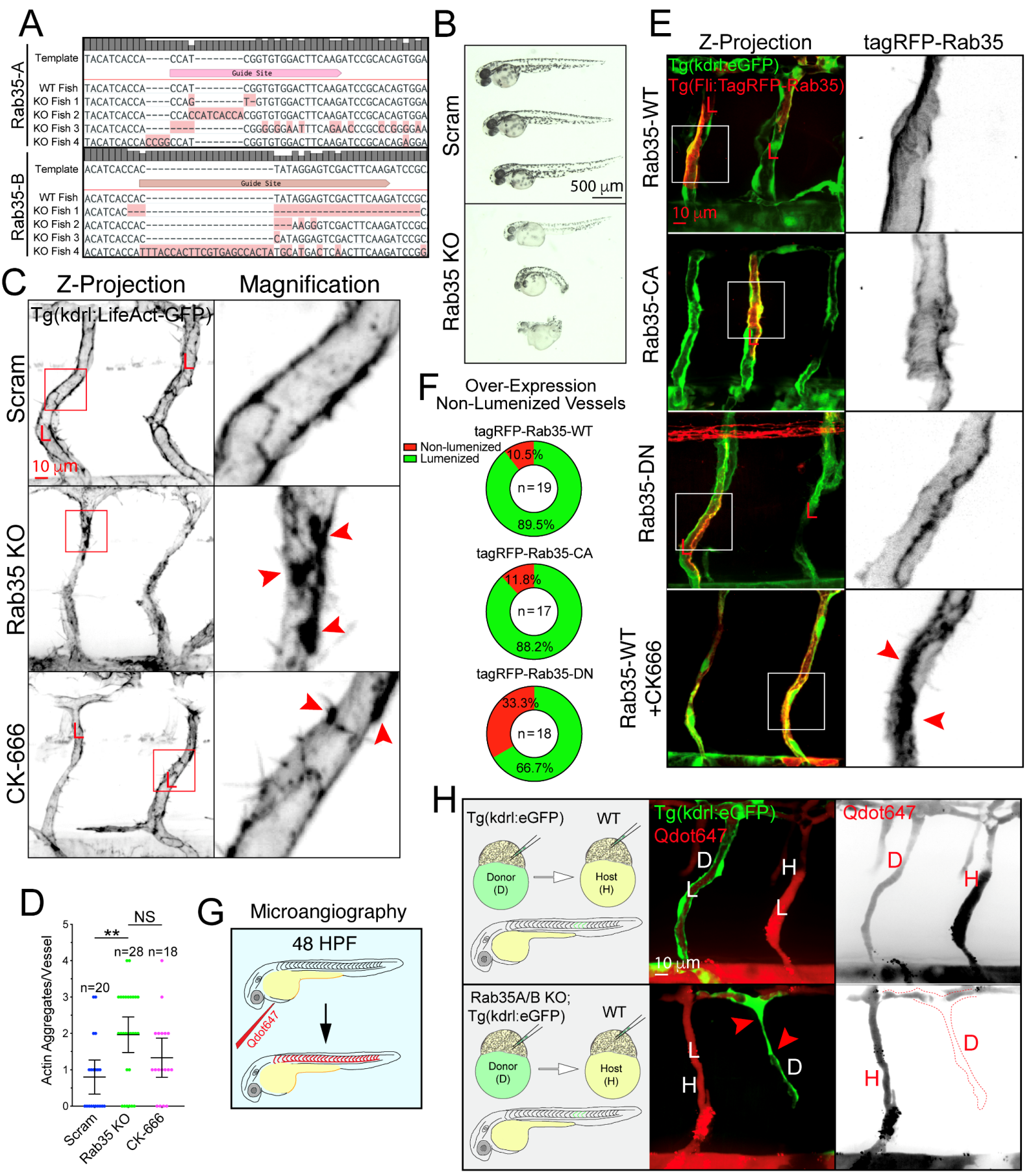

Figure 7. Rab35 is required for blood vessel development in zebrafish. (A) CRISPRmediated knockout of Rab35A/B confirmation by sequencing. Four random fish were sequenced following CRISPR/guide injections. (B) Zebrafish morphology at 48 hours post fertilization (hpf) post injection of scramble (Scram) and Rab35A/B CRISPR guides. (C) Representative images of intersomitic blood vessels (ISVs) of Scram and Rab35A/B knockout as well as CK-666 (Arp Inhibitor) treated zebrafish at $48 \mathrm{hpf}$ expressing endothelial specific LifeAct-GFP. Red arrowheads 
1177 indicate abnormal aggregates of actin. (D) Quantification of actin aggregates between groups. $\mathrm{N}=$ 1178 number of ISVs. A minimum of 5 fish were used per group. (E) Representative images of mosaic 1179 expression of Tag-RFP-Rab35 WT (top row), CA (second row), DN (third row) and WT with CK1180666 treatment in zebrafish at $48 \mathrm{hpf}$. Red arrowheads depict excess of Rab35 at the plasma 1181 membrane. (F) Quantification of non-lumenized vessels at $48 \mathrm{hpf}$ between groups mentioned in panel E. (G) Cartoon representation of microangiography in zebrafish larvae using quantum dots 647 (Qdot647) at $48 \mathrm{hpf}$. (H) Representative images of ISVs after transplantation of Tg(kdrl:GFP) donor (D) into $\mathrm{Tg}(\mathrm{kdrl}$ :mCherry) host $(\mathrm{H})$ (top panels). Bottom panels- representative images of ISVs after transplantation of Rab35A/B knockout donor cells from Tg(kdrl:GFP) line into $\mathrm{Tg}$ (kdrl:mCherry) host. Red arrowheads indicate lumen failure. ${ }^{* *} p<0.01$, NS=Non-Significant. Error bars represent $95 \%$ confidence intervals. Insets are areas of higher magnification. All experiments were done in triplicate. 
A

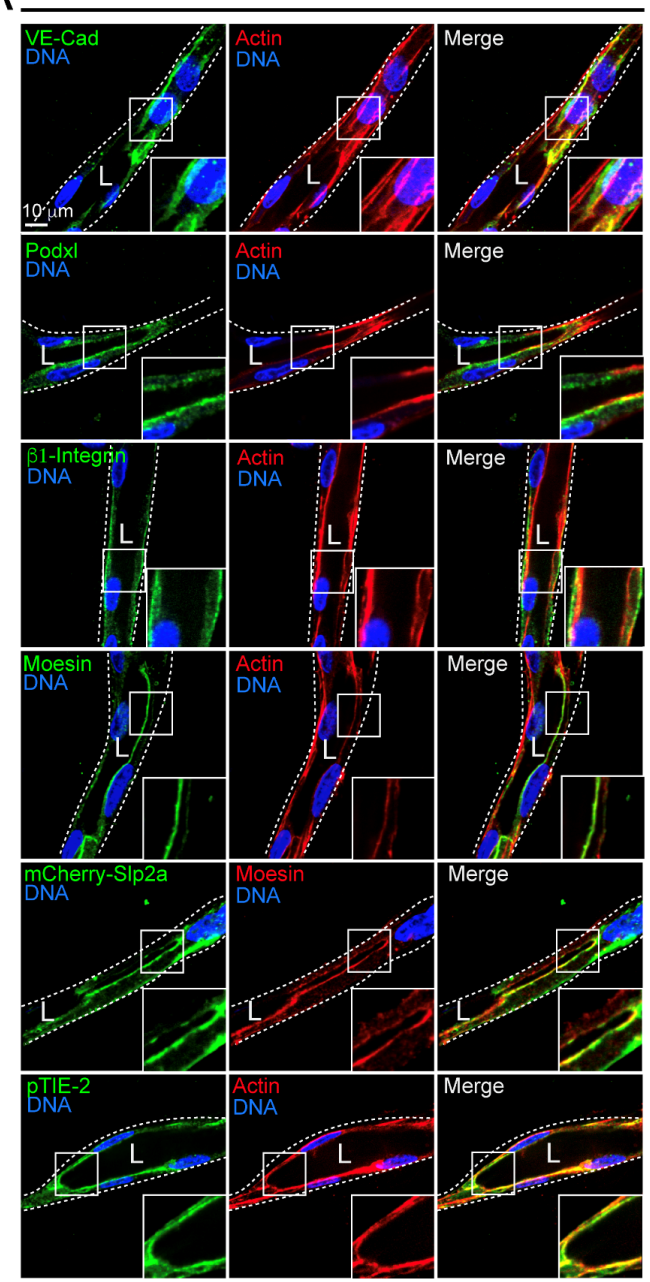

Rab35 si

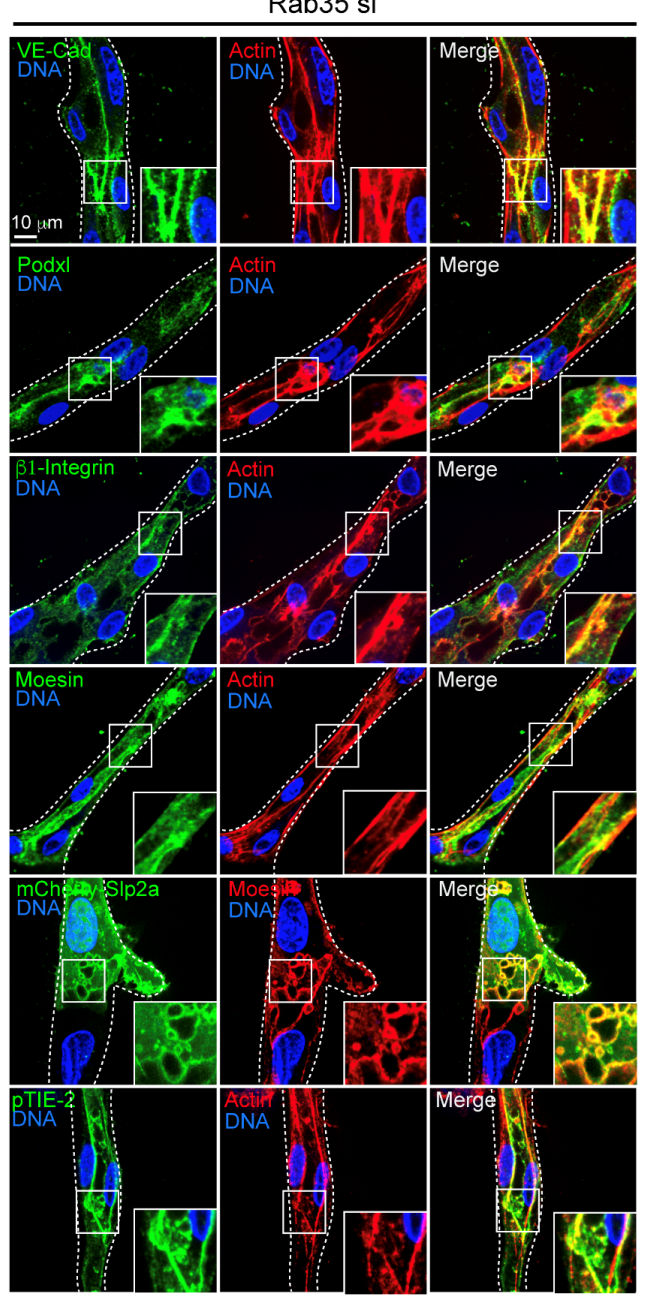

$\mathrm{B}$

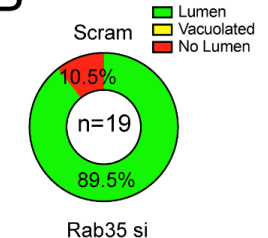

Rab35 si

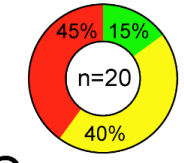

$\left.\mathrm{C}^{8}\right]_{n=34}^{\substack{* \star * * \\ n}}$

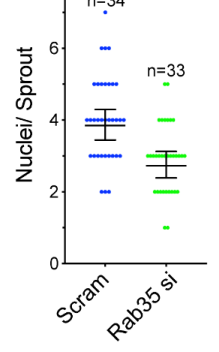

1196

1197

1198

1199

1200

1201

1202

1203

1204

1205

1206

1207

1208

1209

1210

1211

1212

1213
Supplemental Figure 1. Knockdown of Rab35 distorts cell apicobasal polarity. (A) Scramble (Scram) and Rab35 siRNA(si)-treated sprouts stained for VE-cadherin (VE-cad), podocalyxin (Podxl), $\beta 1$-integrin, moesin or phosphorylated Tie2 (pTie2) apical and basal protein markers. Apical marker synaptotagmin-like protein $2 a$ (mCherry-SIp2a) was transduced into sprouts. $L$ denotes lumen and white dotted lines outline sprout exterior. (B) Quantification of lumen formation in Scram and Rab35 siRNA-treated sprouts. Lumens were defined as an open continuous cavity. Vacuolated sprouts were defined as sprouts lacking a contiguous lumen, while exhibiting an excess of large vacuoles. The no lumen group was defined as sprouts that had no visible cavity or vacuoles. N=number of sprouts. (C) Quantification of nuclei per sprout in Scram and Rab35 siRNA treated sprouts. ${ }^{* * *} p<0.001$. Error bars represent $95 \%$ confidence intervals. Insets are areas of higher magnification. All experiments were done using Human umbilical vein endothelial cells in triplicate. 


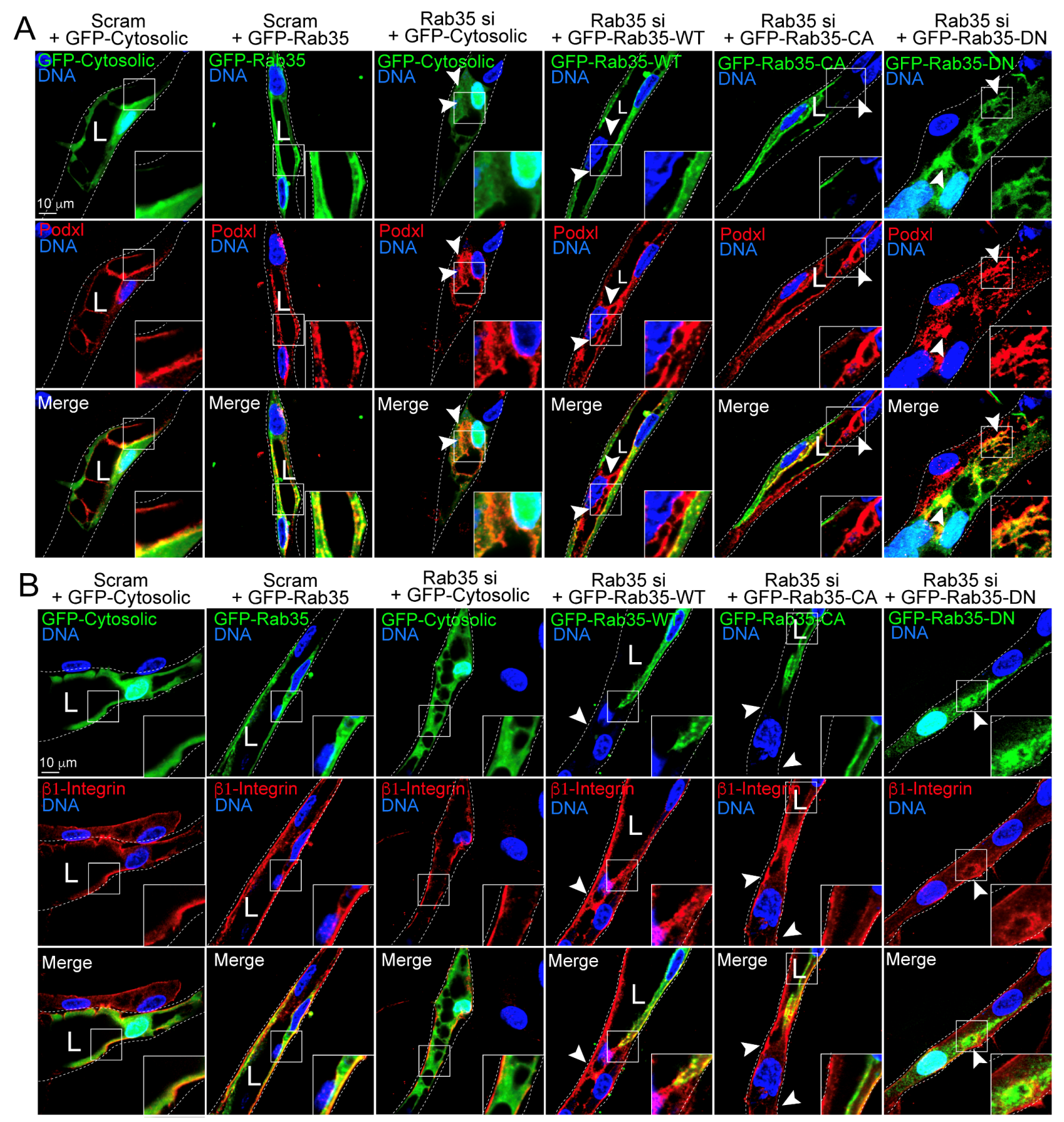

1214

1215

1216

1217

1218

1219

1220

1221

1222

1223

1224
Supplemental Figure 2. Rab35 knockdown disrupts sprout polarity programs. (A,B) Representative images of Rab35 knockdown (KD) sprouts transfected with cytosolic GFP or GFPRab35 wild type (WT), constitutively-active (CA) or dominant negative (DN) for rescues. Sprouts were also stained for apical marker podocalyxin (Podxl) or apical marker $\beta 1$-integrin. Arrowheads denote abnormal localization of podocalyxin or $\beta 1$-integrin. $L$ denotes lumen in all images. White dotted lines mark sprout exterior. Insets are areas of higher magnification. All experiments were done using Human umbilical vein endothelial cells in triplicate. 
A
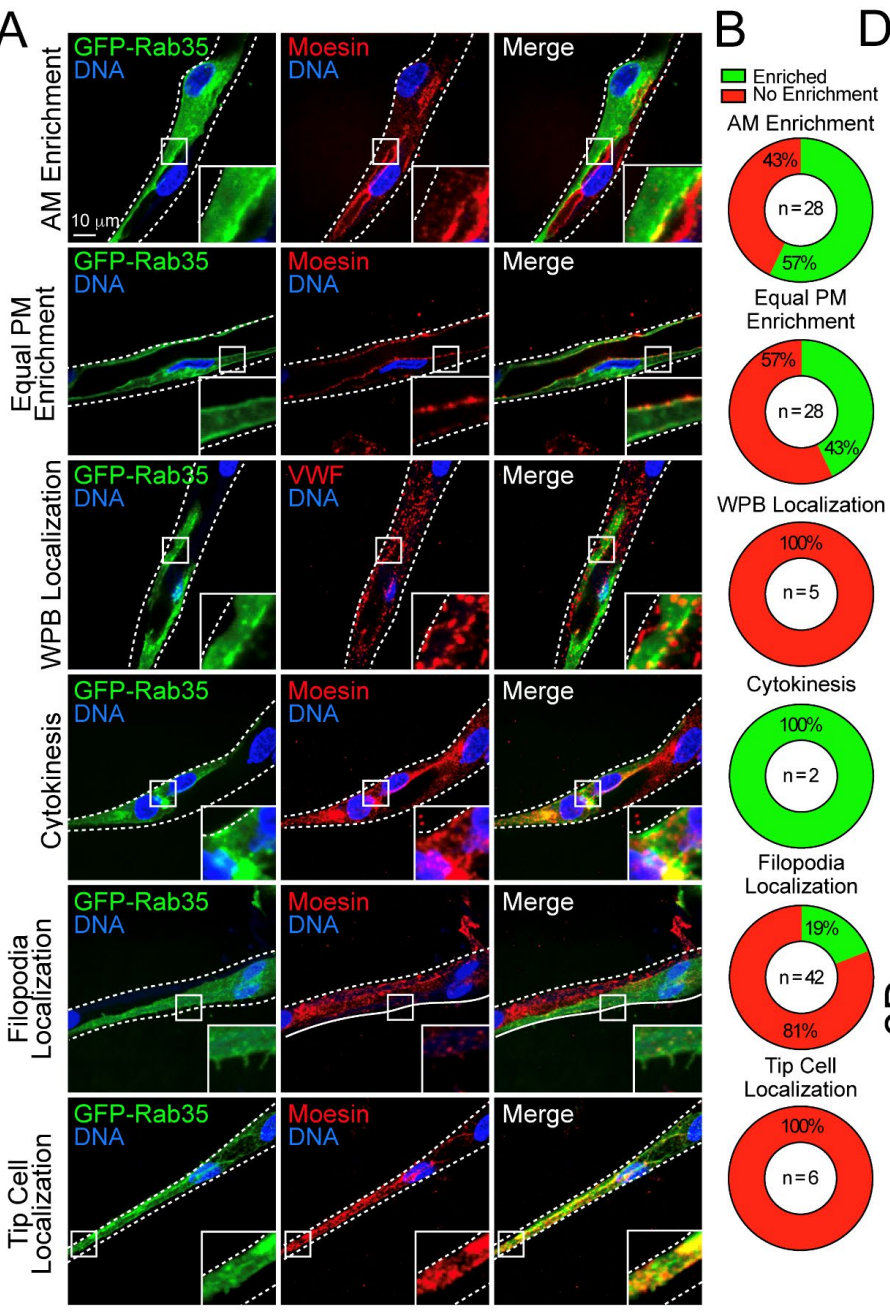

WPB Localization
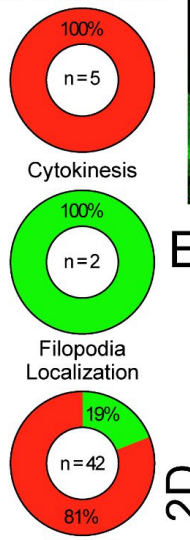

Tip Cell Localization
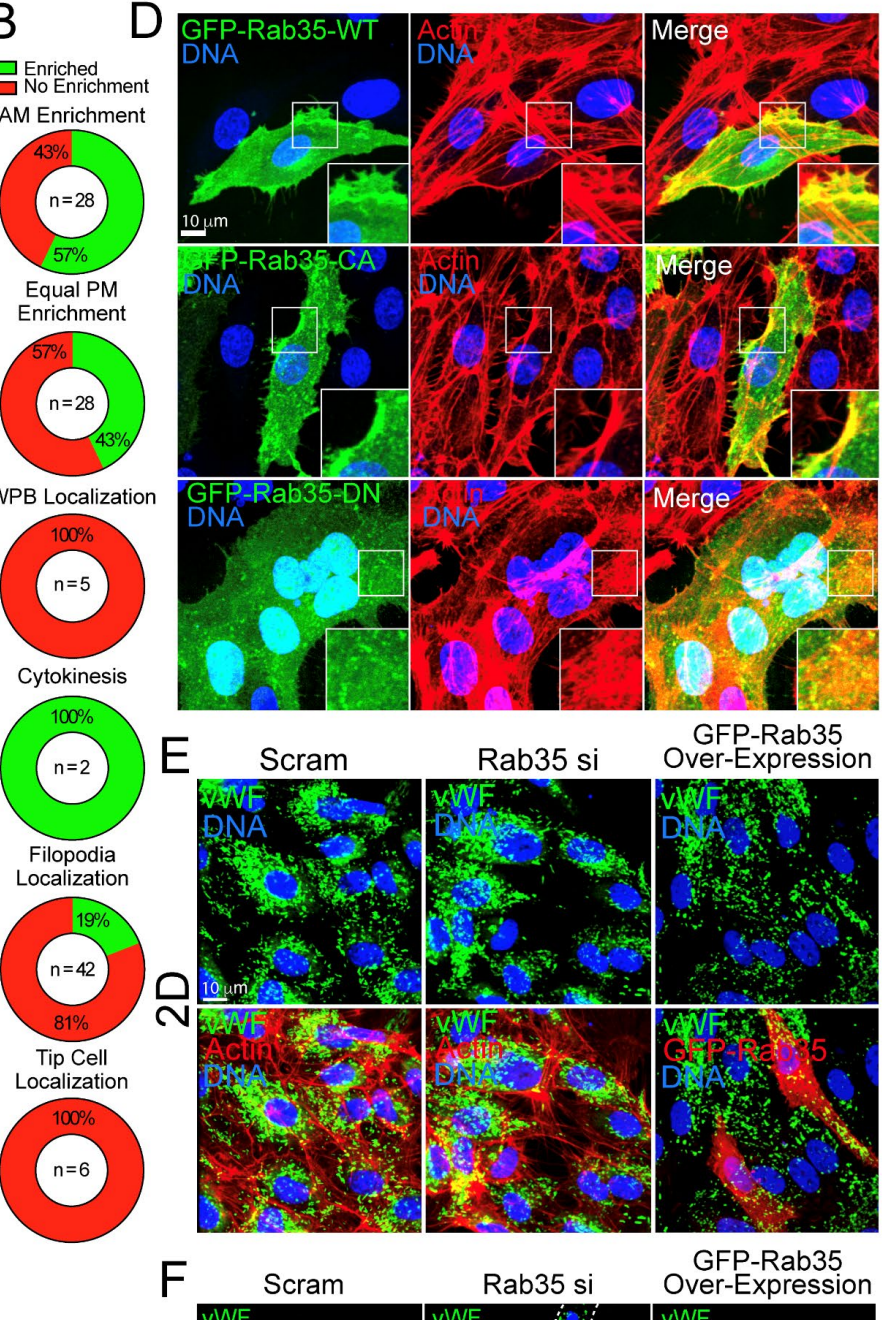

GFP-Rab35

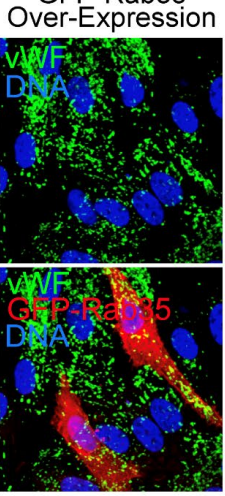

GFP-Rab35
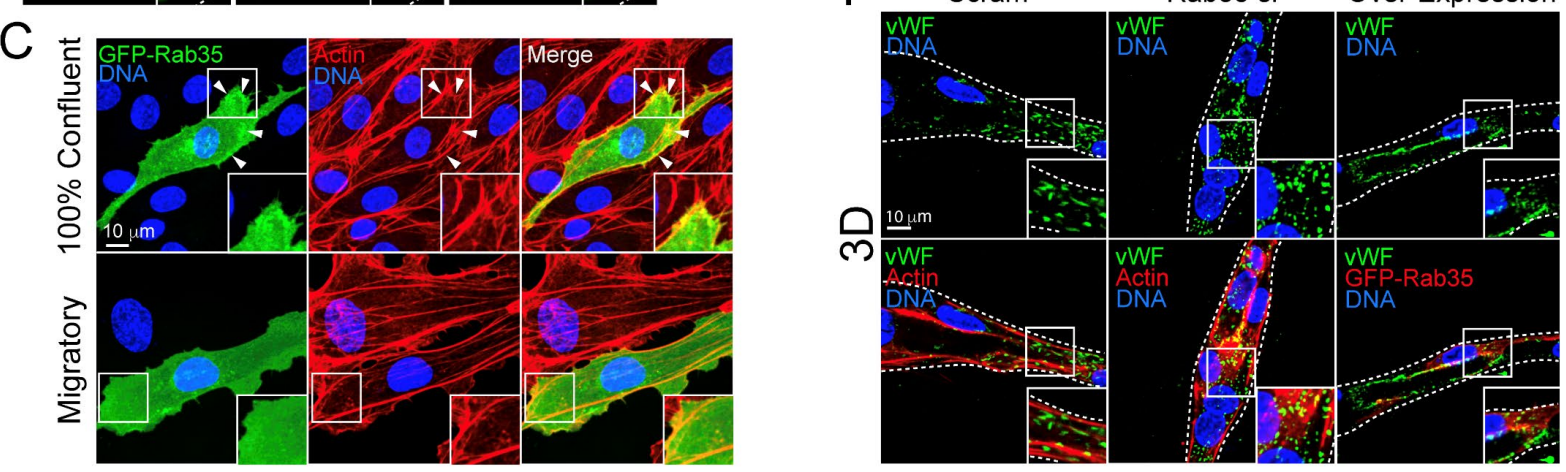

Supplemental Figure 3. Rab35 localizes to the plasma membrane and not to Weibel-Palade

Bodies. (A) Representative images of GFP-Rab35 localization binned by its proximity to the apical plasma membrane (AM), equal enrichment at the basal and apical plasma membrane (equal plasma membrane (PM) enrichment), Weibel-Palade bodies (WPBs), at sites of cytokinesis, filopodia, and most distal cell in the sprout (tip cell). Sprouts were also stained for moesin to mark the apical membrane. (B) Quantification of GFP-Rab35 enrichment with respect to the described conditions in panel A. (C) Representative images of GFP-Rab35 localization in 
2-dimensional culture stain for actin. The top panels are of a confluent monolayer and the bottom panels are of migratory sub-confluent cells. Arrowheads indicate co-localization of actin and GFPRab35. (D) Representative images of 2-dimensional localization of GFP-Rab35 wild type (WT, top panels), constitutively active (CA, middle panels), and dominant negative (DN, bottom panels) stained for actin. (E) Representative images of cells treated with scramble (Scram) or Rab35 siRNA (si) and stained for WPB marker von Willebrand Factor (VWF) and actin or overexpressing GFP-Rab35. (F) Representative images of sprouts treated with Scram or Rab35 siRNA stained for VWF and actin or expressing GFP-Rab35 in 3-dimensional (3D) sprouts. Insets are areas of higher magnification. White dotted lines mark sprout exterior. All experiments were done using Human umbilical vein endothelial cells in triplicate. 

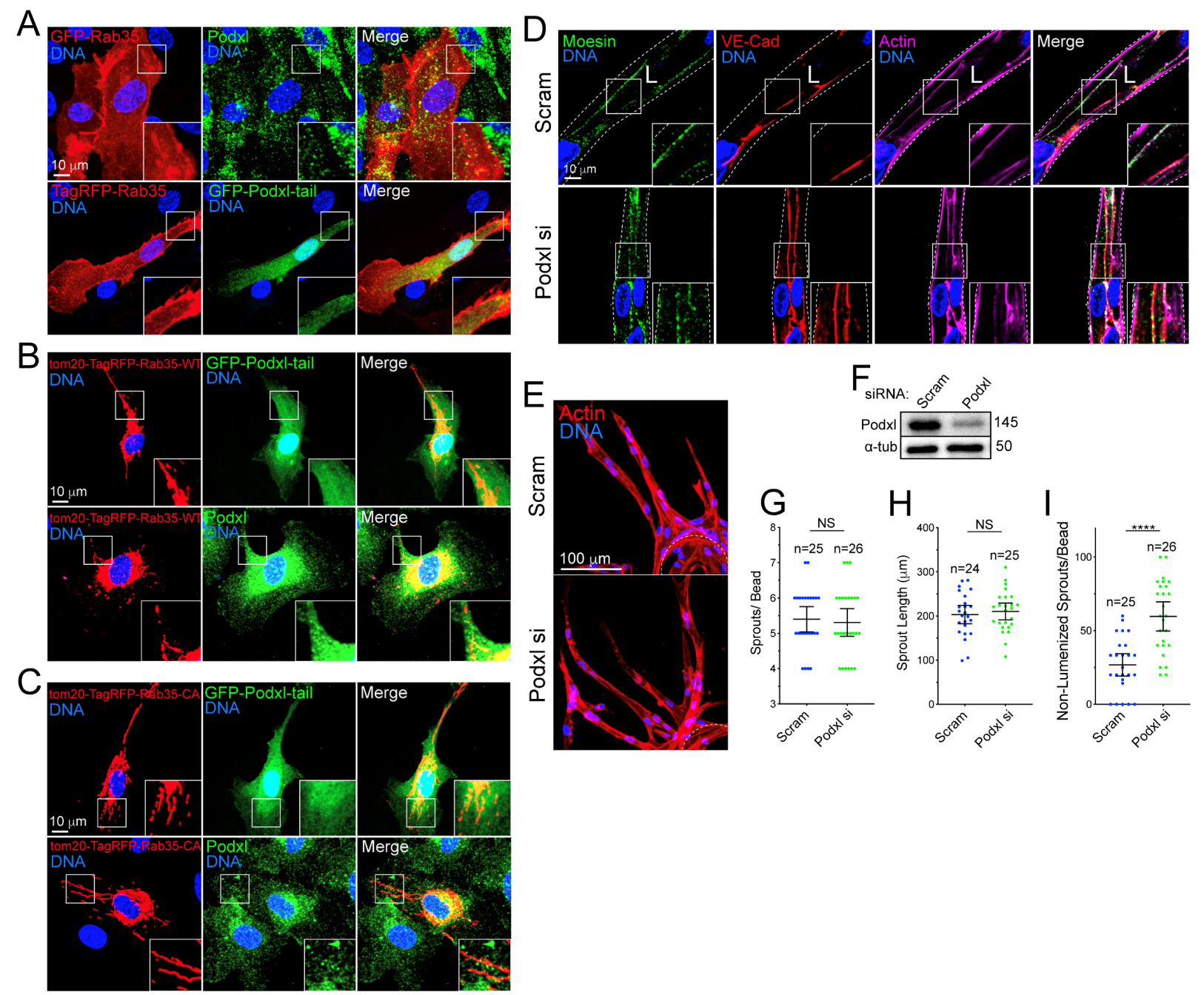

Supplemental Figure 4. Rab35 does not affect podocalyxin trafficking. (A) Two-dimensional localization of GFP-Rab35 with podocalyxin (Podxl) (top panels) and GFP-Podxl-tail (bottom panels). (B). Top panels- cell co-expressing tom20-TagRFP-Rab35- wild type (WT) with GFPPodxl-tail. Bottom panels- cell expressing tom20-TagRFP-Rab35-WT stained for endogenous podocalyxin. (C) Representative image of a cell co-expressing tom20-TagRFP-Rab35constitutively active (CA) mutant with GFP-Podxl-tail. Bottom panels show a cell expressing tom20-tagRFP-Rab35- constitutively active (CA) mutant stained for endogenous podocalyxin. (D) Representative image of sprouts treated with scramble (Scram) or podocalyxin siRNA (si) and stained for moesin, VE-cadherin (VE-cad) and actin. $L$ denotes lumen. White dotted lines mark sprout exterior. (E) Sprout morphology for the same conditions as D. (F) Confirmation of siRNAmediated knockdown by western blot. (G-I) Quantification of indicated sprouting parameters across groups. ${ }^{* * * *} p<0.0001$, NS=Non-Significant. Error bars represent $95 \%$ confidence intervals. Insets are areas of higher magnification. All experiments were done using Human 


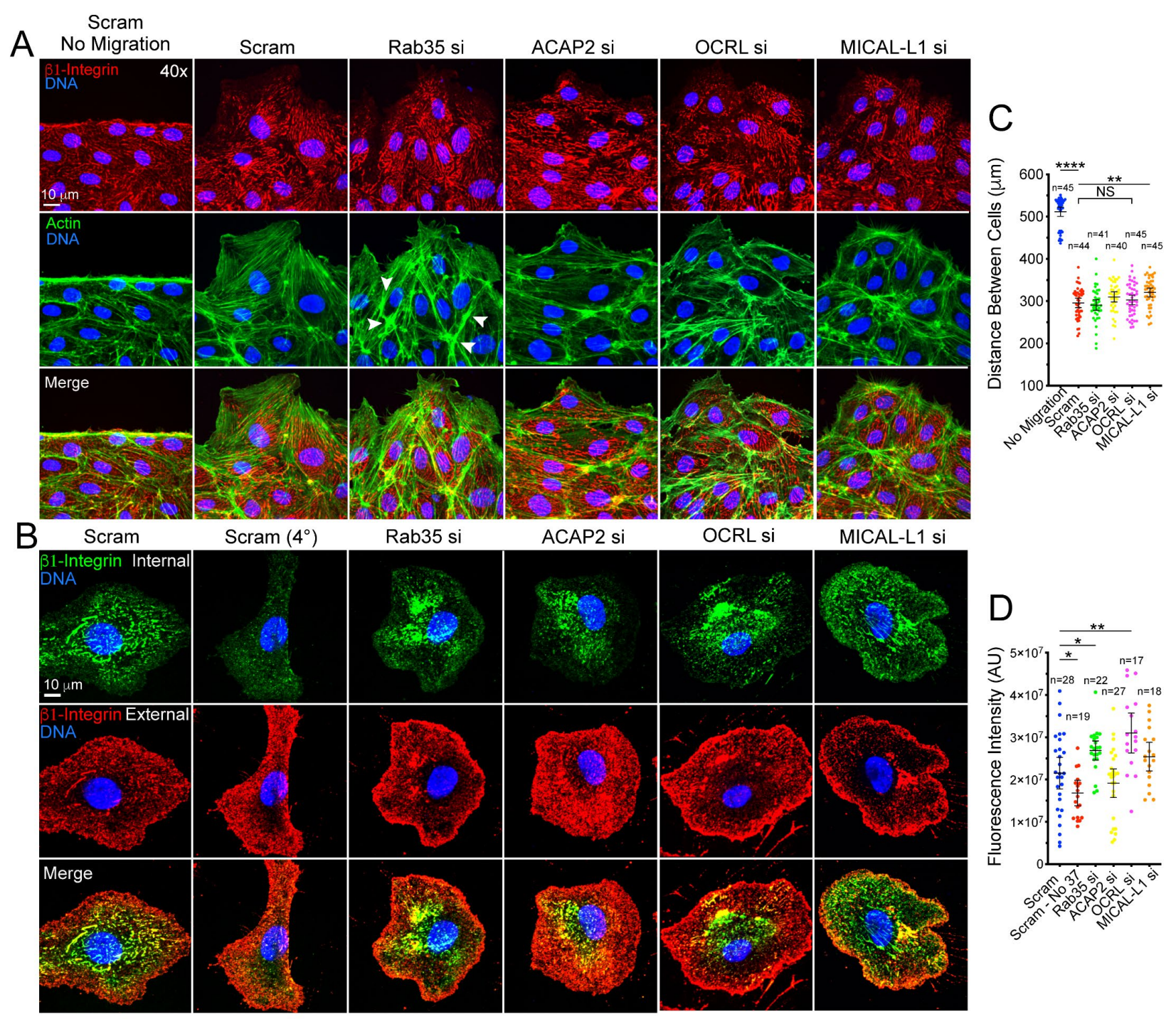
MICAL-L1 siRNA (si). Cells were stained for $\beta 1$-integrin and actin. Arrowheads indicate abnormal actin architecture, namely elevated actin deposition. (B) Antibody feeding assay to test for integrin turnover between conditions. Cells were treated with indicated siRNA. Green channel represents internalized integrins, while the red channel marks only external integrins. As a control to inhibit endocytosis a group was held at $4^{\circ} \mathrm{C}$.

(C) Quantification for the migration assay in A. (D) Fluorescence intensity of internalized $\beta 1$-integrin in panel B. ${ }^{*} p<0.05,{ }^{* *} p<0.01,{ }^{* * * *} p<0.0001$, NS=Non-Significant. Error bars represent $95 \%$ confidence intervals. All experiments were done using Human umbilical vein endothelial cells in triplicate. 

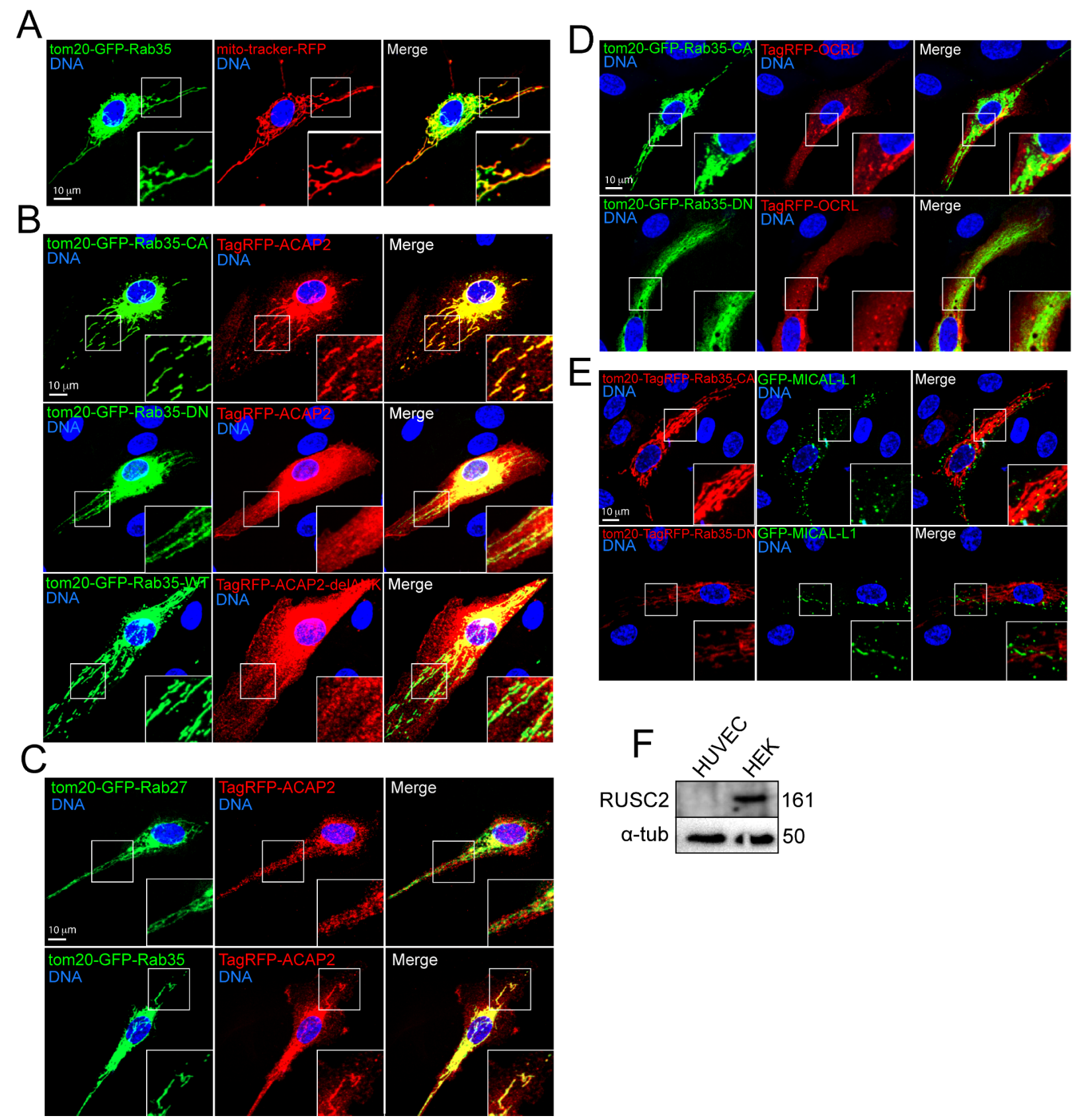

Supplemental Figure 6. Rab35 binds only ACAP2. (A) Cells stained for mitochondria (Mitotracker) and transfected with tom20-tagRFP-Rab35. (B) Representative images of a cell coexpressing tom20-tagRFP-Rab35-wild type (WT), constitutively active (CA), or dominant negative (DN) variants with TagRFP-ACAP2 or ACAP2 with deleted ankyrin repeat domain (delANK). (C) Representative image of a cell expressing tagRFP-ACAP2 and tom20-GFP-Rab27-WT (top panels). Bottom panel is a representative image of a cell expressing of tom20-GFP-Rab35-WT with tagRFP-ACAP2. (D) Top panels- representative image of a cell expressing tom20-GFP- 
1332 GFP-MICAL-L1. Bottom panels- cell expressing tom20-TagRFP-Rab35-DN and GFP-MICAL-L1. (F) Western blot image probing for RUSC2 in both HEK293 cells and Human umbilical vein endothelial cells (HUVECs). Insets are areas of higher magnification. All experiments were done using Human umbilical vein endothelial cells in triplicate. 
A

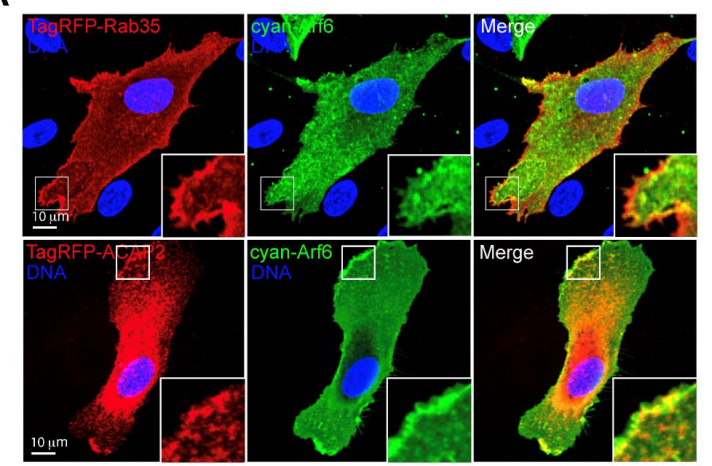

$\mathrm{B}$

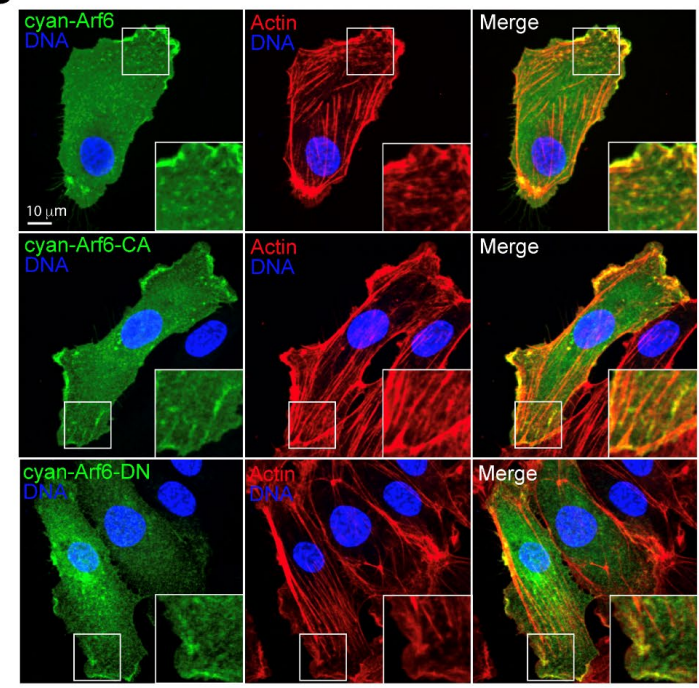

1

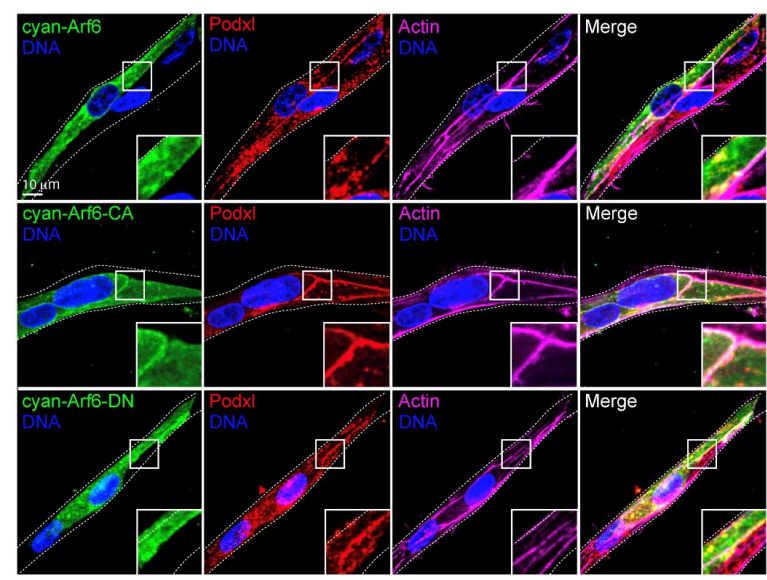

C

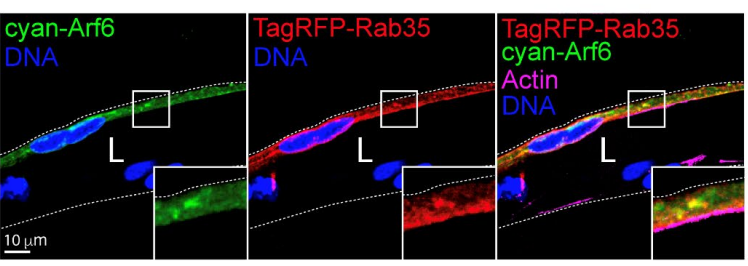

D

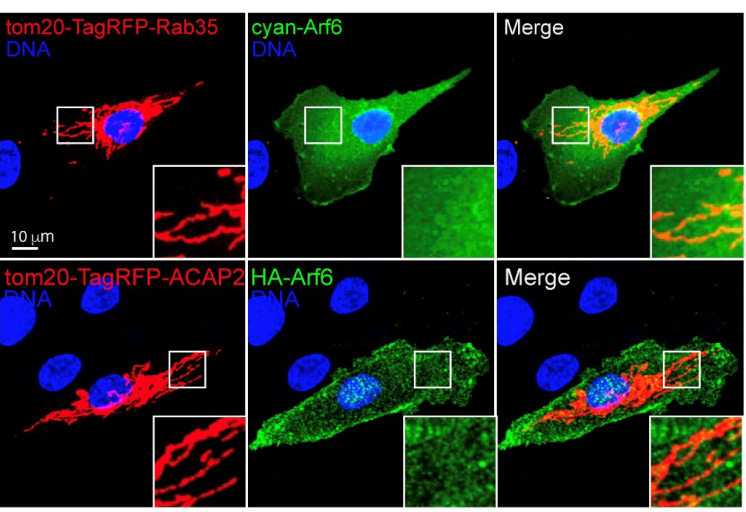

E
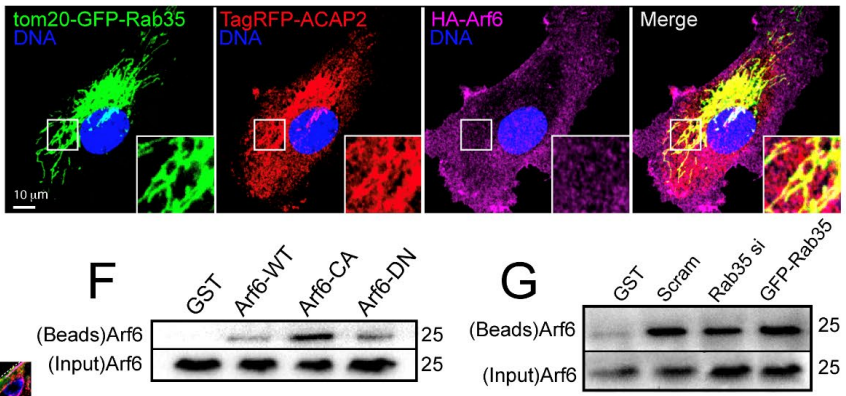

$\mathrm{H}$ Ar6 Expression Lumen Formation

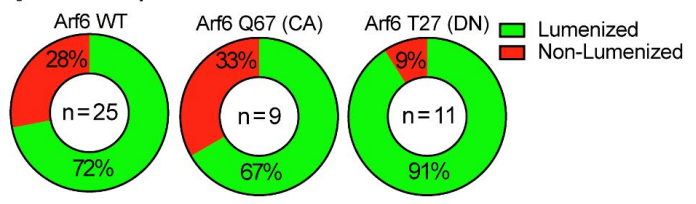

1383

1384

1385

1386

1387

1388

1389

Supplemental Figure 7. Rab35 does not affect Arf6 activity in endothelial cells. (A) Twodimensional localization of cyan-Arf6 with tagRFP-Rab35 (top panels) and ACAP2 (bottom panels). (B) Two-dimensional localization of cyan-Arf6- wild type (WT, top panels), constitutively active (CA, middle panels), and dominant negative (DN, bottom panels) stained for actin. (C) Localization of tag-RFP-Rab35 and cyan-Arf6 in a sprout. (D) Top panel- representative image of 
a cell expressing tom20-tagRFP-Rab35 and cyan-Arf6. Bottom panel- representative image of a cell expressing tom20-tagRFP-ACAP2 and HA-Arf6. (E) Representative image of a cell expressing tom20-GFP-Rab35, tagRFP-ACAP2 and HA-Arf6. (F) Pulldown assay using GGA3 to probe for activated Arf6. Cells were transfected with WT, CA, or DN Arf6. (G) Pulldown assay using GGA3 to probe for activated Arf6. Cells were treated with scramble (Scram) and Rab35 siRNA (si) or transfected with GFP-Rab35. () Quantification of open or collapsed lumens after transfection with WT, CA, or DN cyan-Arf6. N= number of sprouts. (I) Representative images of sprouts transduced with WT, CA, or DN cyan-Arf6 stained for Podocalyxin (Podxl) and actin. L denotes lumen in all images. White dotted lines mark sprout exterior. Insets are areas of higher magnification. All experiments were done using Human umbilical vein endothelial cells in triplicate. 

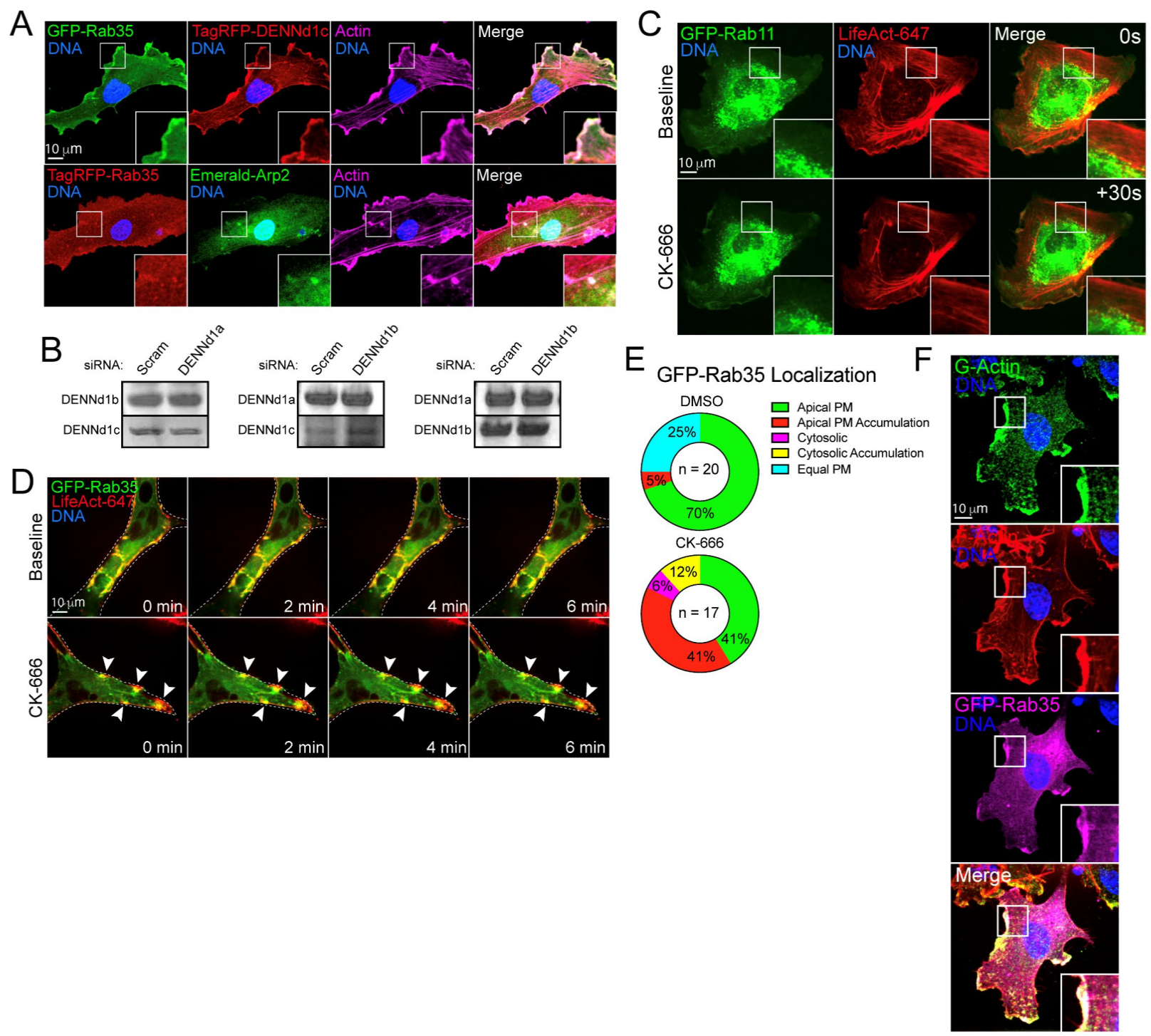
panel- representative image of a cell expressing GFP-Rab35 and tagRFP-DENNd1c. Bottom panel- representative image of a cell expressing GFP-Rab35 and Emerald-Arp2. (B) Western blot of DENNd1a-c knockdown. For each blot a DENNd1 was knocked down and the remaining two DENND1s were probed for to test for compensation effects. (C) Representative live-image of a cell expressing GFP-Rab11 and TagRFP647 (647)-LifeAct before and after CK-666 treatment. (D) GFP-Rab35 and LifeAct-647 co-expression in sprout live-imaged at baseline and following treatment with CK-666. Arrowheads indicate accumulations of GFP-Rab35 and LifeAct-647. Dotted line indicates sprout exterior. (E) Quantification of GFP-Rab35 localization upon DMSO (vehicle) or CK-666 administration. Apical plasma membrane (PM, uniformly localized to apical membrane), apical PM accumulation (Rab35 puncta at the apical membrane), cytosolic (localized 
1458 in the cytoplasm), cytosolic accumulations (Rab35 puncta in the cytoplasm), equal PM (Rab35 1459 equally distributed between the apical and basal membranes). Two-dimensional localization of 1460 GFP-Rab35 with globular-actin and filamentous-actin. (F) Representative image of a cell 1461 expressing GFP-Rab35 and stained for filamentous (F) and globular (G) actin. Insets are areas of higher magnification. All experiments were done using Human umbilical vein endothelial cells 


\section{MOVIE FIGURE LEGENDS}

1469 Movie 1. Sprout expressing GFP-Rab35. L denotes lumen.

1471 Movie 2. Sprout expressing GFP-Rab35 and LifeAct-TagRFP647 (FarRed) treated with

1472 scrambled siRNA. L denotes lumen.

1473

1474 Movie 3. Sprout expressing GFP-Rab35 and LifeAct-TagRFP647 (FarRed) treated with

1475 DENNd1c siRNA. L denotes lumen. Arrow marks actin accumulation.

Movie 4. Sprout expressing GFP-Rab35 and LifeAct-TagRFP647 (FarRed) treated with DMSO.

1478 Arrow marks normal actin buttressing at junctions.

Movie 5. Sprout expressing GFP-Rab35 and LifeAct-TagRFP647 (FarRed) treated with CK666. Arrow marks actin accumulations.

1483 Movie 6. Cell expressing TagRFP-Rab35 and GFP-LifeAct before and after CK-666 treatment.

1485 Movie 7. Cell expressing TagRFP-DENNd1c and GFP-LifeAct before and after CK-666 1486 treatment.

1488 Movie 8. Cell expressing mCherry-Arp2 and GFP-Rab35 before and after CK-666 treatment.

1490 Movie 9. Cell expressing GFP-Rab35, LifeAct-TagRFP647 (FarRed), and ligand-modulated 1491 antibody fragments targeted to the mitochondria (mito-LAMA) before and after trimethoprim 1492 (TMP) administration. 
1495 Movie 10. Cell expressing GFP-Rab35, mCherry-Arp2, and ligand-modulated antibody

1496 fragments targeted to the mitochondria (mito-LAMA) before and after trimethoprim (TMP)

1497 administration.

Movie 11. Cell expressing GFP-Rab35, TagRFP-DENNd1c, and ligand-modulated antibody

1500 fragments targeted to the mitochondria (mito-LAMA) before and after trimethoprim (TMP)

1501 administration.

1502

1503 Movie 12. Cell expressing GFP-Rab35, mCherry-Arp2, and ligand-modulated antibody

1504 fragments targeted to the mitochondria (mito-LAMA) before and after trimethoprim (TMP)

1505 administration. After TMP treatment cell were also treated with CK-666.

1506

1507 Movie 13. DENNd1c knockdown (siRNA) cell expressing GFP-Rab35, mCherry-Arp2, and

1508 ligand-modulated antibody fragments targeted to the mitochondria (mito-LAMA) before and after

1509 trimethoprim (TMP) administration. 
1510 MAJOR RESOURCE TABLE

\begin{tabular}{|c|c|c|}
\hline Reagent & Vendor & Catalog \# \\
\hline $\begin{array}{l}\text { OPTI-MEM } 1 \text { Reduced Serum } \\
\text { Medium, no phenol red }\end{array}$ & ThermoFisher & 31985070 \\
\hline $\begin{array}{l}\text { Polyethyleneamine Branched } \\
\text { (PEI) }\end{array}$ & Sigma-Aldrich & 408727 \\
\hline $\begin{array}{l}\text { Chloroquine Diphosphate } \\
\text { Crystalline (CQ) }\end{array}$ & Sigma-Aldrich & C6628-25G \\
\hline $\begin{array}{l}\text { Endothelial Cell Growth } \\
\text { Medium } 2\end{array}$ & PromoCell & C-22011 \\
\hline $\begin{array}{l}\text { DMEM, High Glucose, with L- } \\
\text { Glutamine }\end{array}$ & Genesee Scientific & $25-500$ \\
\hline $\begin{array}{l}\text { GenClone Fetal Bovine } \\
\text { Serum (FBS) }\end{array}$ & Genesee Scientific & $25-514$ \\
\hline $\begin{array}{l}\text { Penicillin-Streptomycin 100X } \\
\text { Solution }\end{array}$ & Genesee Scientific & P4333-100ML \\
\hline $\begin{array}{l}\text { DPBS, no Calcium, no } \\
\text { Magnesium }\end{array}$ & ThermoFisher & 14190250 \\
\hline $\begin{array}{l}\text { Trypsin-EDTA, o.25\% 1X, } \\
\text { phenol red }\end{array}$ & Genesee Scientific & $25-510$ \\
\hline $\begin{array}{l}\text { Paraformaldahyde } 20 \% \\
\text { Aqueous Sol. EM Grade }\end{array}$ & Electron Microscopy Sciences & 15713 \\
\hline $\begin{array}{l}\text { BSA Lyophilized Powder, } \\
\text { Fraction V }\end{array}$ & Genesee Scientific & $25-529$ \\
\hline $\begin{array}{l}\text { Cytoskeleton G actin/ F actin } \\
\text { In Vivo Assay Kit }\end{array}$ & Cytoskeleton, Inc. & ВK037-ВK037 \\
\hline $\begin{array}{l}\text { Culture-Insert } 2 \text { Well in } \mu \text {-Dish } \\
35\end{array}$ & Ibidi & 81176 \\
\hline Dimethyl Sulfoxide (DMSO) & Sigma-Aldrich & D2650-5X10ML \\
\hline $\begin{array}{l}\text { Silencer }{ }^{\mathrm{TM}} \text { Negative Control } \\
\text { No. } 1 \text { siRNA }\end{array}$ & ThermoFisher & AM4611 \\
\hline Rab35 siRNA & ThermoFisher & siRNA ID: s21709 \\
\hline ACAP2 siRNA & ThermoFisher & siRNA ID: s24011 \\
\hline OCRL SiRNA & ThermoFisher & siRNA ID: s9819 \\
\hline MICAL-L1 siRNA & Thermo Scientific & siRNA ID: s39940 \\
\hline
\end{tabular}




\begin{tabular}{|c|c|c|}
\hline RUSC2 SiRNA & Thermo Scientific & siRNA ID: s19070 \\
\hline Podxl siRNA & Thermo Scientific & siRNA ID: s10771 \\
\hline DENNd1a & Thermo Scientific & siRNA ID: s33637 \\
\hline DENNd1b & Thermo Scientific & siRNA ID: s29140 \\
\hline DENNd1c & Thermo Scientific & siRNA ID: s36719 \\
\hline Cytodex Microcarrier Beads & Sigma-Aldrich & C3275-10G \\
\hline CK-666 & Sigma-Aldrich & SML0006-5MG \\
\hline Trimethoprim (TMP) & Sigma-Aldrich & T7883-5G \\
\hline NP-G2-044 (Fascin Inhibitor) & Selleck Chem & S2962 \\
\hline GGA3 PBD-Beads & Cytoskeleton, Inc. & GGA07-A \\
\hline $\begin{array}{l}\text { High Capacity Reverse } \\
\text { Transcription Kit }\end{array}$ & ThermoFisher & 4368814 \\
\hline $\begin{array}{l}\text { Fibrinogen Type 1-S from } \\
\text { Bovine Plasma }\end{array}$ & Sigma-Aldrich & F8630-1G \\
\hline $\begin{array}{l}\text { Thrombin from Bovine } \\
\text { Plasma }\end{array}$ & Sigma-Aldrich & T7513-500UN \\
\hline Aprotinin Protease Inhibitor & ThermoFisher & 78432 \\
\hline $\begin{array}{l}\text { Phenol-Red (Zebrafish } \\
\text { Injection Mixture) }\end{array}$ & Avantor/ VWR & $34487-61-1$ \\
\hline CRIPSR gRNA & $\begin{array}{l}\text { Integrated DNA Technologies } \\
\text { (IDT) }\end{array}$ & \\
\hline $\begin{array}{l}\text { Alt-R® S.p. Cas9 Nuclease } \\
\text { V3, } 100 \mu \mathrm{g}\end{array}$ & $\begin{array}{l}\text { Integrated DNA Technologies } \\
\text { (IDT) }\end{array}$ & 1081058 \\
\hline CellTracker Deep Red & ThermoFisher & M22426 \\
\hline $\begin{array}{l}\text { 3-Aminobenzoic Acid Ethyl } \\
\text { Ester (Tricaine) }\end{array}$ & Sigma-Aldrich & A5040-25G \\
\hline $\begin{array}{l}\text { Latex Beads, Polystyrene } \\
\text { Carboxylate Mod }\end{array}$ & Sigma-Aldrich & L3280-1ML \\
\hline $\begin{array}{l}\text { Dynabeads }{ }^{\mathrm{TM}} \text { Protein } \mathrm{G} \text { for } \\
\text { Immunoprecipitation }\end{array}$ & ThermoFisher & 10003D \\
\hline MitoTracker DeepRed & ThermoFisher & M22426 \\
\hline Trizol Reagent & ThermoFisher & 15596026 \\
\hline
\end{tabular}




\begin{tabular}{|c|c|c|}
\hline Chloroform & Sigma-Aldrich & 288306 \\
\hline $\begin{array}{l}\text { MEGAscript }{ }^{\mathrm{TM}} \mathrm{T3} \\
\text { Transcription Kit }\end{array}$ & ThermoFisher & AM1338 \\
\hline BCA Protein Assay Kit & ThermoFisher & 23225 \\
\hline NHLF & Lonza & CC-2512 \\
\hline HEK 293-A & ThermoFisher & R70507 \\
\hline Microcarrier beads & Amersham & $17-0485-01$ \\
\hline Protease inhibitor cocktail & GoldBio & GB-334-20 \\
\hline Agarose Resin & GoldBio & G-250-G \\
\hline $\begin{array}{l}\text { Fura Red }{ }^{\mathrm{TM}}, \mathrm{AM} \text {, cell } \\
\text { permeant }\end{array}$ & ThermoFisher & F3020 \\
\hline
\end{tabular}

\section{ANTIBODIES}

\begin{tabular}{|l|l|l|l|}
\hline Target Antigen & $\begin{array}{l}\text { Vendor or } \\
\text { Source }\end{array}$ & $\begin{array}{l}\text { Catalog No.I } \\
\text { Clone }\end{array}$ & $\begin{array}{l}\text { Working } \\
\text { Concentration }\end{array}$ \\
\hline Rab35 & ThermoFisher & PA531674 & $1: 500(\mathrm{WB})$ \\
\hline ACAP2 & ThermoFisher & PA557069 & $1: 500(\mathrm{WB})$ \\
\hline OCRL & ThermoFisher & PA527844 & $1: 200(\mathrm{WB})$ \\
\hline MICAL-L1 & ThermoFisher & PA5107177 & $1: 200(\mathrm{WB})$ \\
\hline RUSC2 & ThermoFisher & PA572752 & $1: 200(\mathrm{WB})$ \\
\hline Arf6 & Santa Cruz & Sc-7971 & $1: 200(\mathrm{WB})$ \\
\hline Myc-tag & ThermoFisher & 132500 & $1: 1000(\mathrm{IHC})$ \\
\hline HA-tag & ThermoFisher & 26183 & $1: 1000(\mathrm{IHC})$ \\
\hline cyan & Bio-Rad & AHP2986 & $1: 1000(\mathrm{IHC})$ \\
\hline Alpha-tubulin & Abcam & ab52866 & $\begin{array}{l}0.0648 \mathrm{ug} / \mathrm{mL} \\
(1: 10,000)(\mathrm{WB})\end{array}$ \\
\hline
\end{tabular}




\begin{tabular}{|c|c|c|c|}
\hline GAPDH & ThermoFisher & PA1988 & 1:1000 (WB) \\
\hline Moesin & Abcam & $a b 52490$ & $\begin{array}{l}0.05 \mathrm{ug} / \mathrm{mL} \\
(1: 1000)(\mathrm{IHC})\end{array}$ \\
\hline VE-Cadherin & ThermoFisher & $14-1441-82$ & $\begin{array}{l}0.5 \mathrm{ug} / \mathrm{mL} \\
(1: 1000)(\mathrm{IHC})\end{array}$ \\
\hline Podocalyxin & R\&D & AF1658 & $\begin{array}{l}15 \mathrm{ug} / \mathrm{mL}(1: 200) \\
\text { (WB \& IHC) }\end{array}$ \\
\hline $\begin{array}{l}\text { Von Willebrand } \\
\text { Factor }\end{array}$ & Abcam & ab6994 & $\begin{array}{l}\text { 10ug/mL } \\
(1: 1000)(\mathrm{IHC})\end{array}$ \\
\hline$\beta 1-$ Integrin & Abcam & ab30394 & $1: 500(\mathrm{IHC})$ \\
\hline $\begin{array}{l}\text { Phosphorylated } \\
\text { TIE-2/TEK } \\
\text { (Tyr992) }\end{array}$ & Sigma Aldrich & ABF131 & $\begin{array}{l}0.25 \mathrm{ug} / \mathrm{mL} \\
(1: 500)(\mathrm{IHC})\end{array}$ \\
\hline $\begin{array}{l}\text { Anti-HA-Tag, } \\
\text { Rabbit } \\
\text { Monoclonal }\end{array}$ & Sigma-Aldrich & $\begin{array}{l}\text { SAB5600116- } \\
\text { 100UG }\end{array}$ & $5 \mathrm{ug} / \mathrm{mL}$ \\
\hline $\begin{array}{l}\text { Alexa Fluor }{ }^{\mathrm{TM}} \\
488 \text { Phalloidin }\end{array}$ & ThermoFisher & A12379 & 1 uM (1:200) \\
\hline $\begin{array}{l}\text { Alexa Fluor }{ }^{\mathrm{TM}} \\
647 \text { Phalloidin }\end{array}$ & ThermoFisher & A22287 & $1 \mathrm{uM}(1: 200)$ \\
\hline $\begin{array}{l}\text { Alexa Fluor }{ }^{\mathrm{TM}} \\
555 \text { Phalloidin }\end{array}$ & ThermoFisher & A34055 & 1 uM $(1: 200)$ \\
\hline $\begin{array}{l}\text { Goat anti-Rabbit } \\
\text { IgG }(\mathrm{H}+\mathrm{L}) \\
\text { Secondary } \\
\text { Antibody, Alexa } \\
\text { Fluor } 488\end{array}$ & ThermoFisher & A11008 & $1 \mathrm{ug} / \mathrm{mL}(1: 500)$ \\
\hline $\begin{array}{l}\text { Donkey anti- } \\
\text { Rabbit lgG }(\mathrm{H}+\mathrm{L}) \\
\text { Secondary } \\
\text { Antibody, Alexa } \\
\text { Fluor } 555\end{array}$ & ThermoFisher & A31572 & 1ug/mL (1:500) \\
\hline $\begin{array}{l}\text { Donkey anti-goat } \\
\operatorname{lgG}(\mathrm{H}+\mathrm{L}) \\
\text { Secondary } \\
\text { Antibody, Alexa } \\
\text { Flour } 488\end{array}$ & ThermoFisher & A11055 & $1 \mathrm{ug} / \mathrm{mL}(1: 500)$ \\
\hline
\end{tabular}


1515

1516

1517

1518

1519

\begin{tabular}{|l|l|l|l|}
\hline $\begin{array}{l}\text { Donkey anti- } \\
\text { Goat IgG }(\mathrm{H}+\mathrm{L}) \\
\text { Cross-Adsorbed } \\
\text { Secondary } \\
\text { Antibody, Alexa } \\
\text { Fluor 555 }\end{array}$ & ThermoFisher & A21432 & $1 \mathrm{ug} / \mathrm{mL}(1: 500)$ \\
\hline $\begin{array}{l}\text { Chicken anti- } \\
\text { Rabbit IgG (H+L) } \\
\text { Cross-Adsorbed } \\
\text { Secondary } \\
\text { Antibody, Alexa } \\
\text { Fluor 647 }\end{array}$ & ThermoFisher & A21443 & $1 \mathrm{ug} / \mathrm{mL}(1: 500)$ \\
\hline $\begin{array}{l}\text { Goat Anti-Rabbit } \\
\text { HRP }\end{array}$ & $\begin{array}{l}\text { Genesee } \\
\text { Scientific }\end{array}$ & $20-303$ & \\
\hline
\end{tabular}

\section{OLIGOS AND SGRNA}

\begin{tabular}{|l|l|l|}
\hline Name & Sequence & Function \\
\hline Rab35a crRNA & CCATCGGTGTGGACTTCAAG & sgRnA Target \\
\hline Rab35b crRNA & CTATAGGAGTCGACTTCAAG & sgRNA Target \\
\hline Rab35a_seqF & $\begin{array}{l}\text { GCCAATCAGATTCGAGATCCAG } \\
\text { AC }\end{array}$ & Sequencing Primer \\
\hline Rab35a_seqR & $\begin{array}{l}\text { CACTCACGTGGAGGTGATTGTCC } \\
\text { TG }\end{array}$ & Sequencing Primer \\
\hline Rab35b_seqF & CACGCATAGTTCAATGGTGTGTG & Sequencing Primer \\
\hline Rab35b_seqR & $\begin{array}{l}\text { GCACACCCCTATCATGACACTAC } \\
\text { TC }\end{array}$ & Sequencing Primer \\
\hline
\end{tabular}

